\title{
Frobenius Structures Over Hilbert C*-Modules
}

\author{
Chris Heunen $^{1}$ (D), Manuel L. Reyes ${ }^{2}$ (I) \\ 1 School of Informatics, University of Edinburgh, Edinburgh, UK. E-mail: chris.heunen@ed.ac.uk \\ 2 Department of Mathematics, Bowdoin College, Brunswick, USA. E-mail: reyes@ bowdoin.edu
}

Received: 5 June 2017 / Accepted: 9 April 2018

Published online: 9 June 2018 - (C) The Author(s) 2018

\begin{abstract}
We study the monoidal dagger category of Hilbert $\mathrm{C}^{*}$-modules over a commutative $\mathrm{C}^{*}$-algebra from the perspective of categorical quantum mechanics. The dual objects are the finitely presented projective Hilbert $\mathrm{C}^{*}$-modules. Special dagger Frobenius structures correspond to bundles of uniformly finite-dimensional $\mathrm{C}^{*}$-algebras. A monoid is dagger Frobenius over the base if and only if it is dagger Frobenius over its centre and the centre is dagger Frobenius over the base. We characterise the commutative dagger Frobenius structures as finite coverings, and give nontrivial examples of both commutative and central dagger Frobenius structures. Subobjects of the tensor unit correspond to clopen subsets of the Gelfand spectrum of the $\mathrm{C}^{*}$-algebra, and we discuss dagger kernels.
\end{abstract}

\section{Introduction}

Categorical quantum mechanics [36] provides a powerful graphical calculus for quantum theory. It achieves this by stripping the traditional Hilbert space model of much detail. Nevertheless, the main examples remain based on Hilbert spaces, and relations between sets. The latter can be extended to take scalars in arbitrary quantales [2]. This article extends scalars in the former from complex numbers to arbitrary commutative $\mathrm{C}^{*}$ algebras. In other words, we study the monoidal category of Hilbert modules over a commutative $\mathrm{C}^{*}$-algebra. This provides a genuinely new model, that is interesting for various reasons.

- Just like commutative $\mathrm{C}^{*}$-algebras are dual to locally compact Hausdorff spaces, we prove that Hilbert modules are equivalent to bundles of Hilbert spaces over locally compact Hausdorff spaces (in Sect. 4). Instead of a single Hilbert space of states, we may have Hilbert spaces over every point of a base space that vary continuously.

- We prove that the abstract scalars hide more structure than previously thought: subobjects of the tensor unit correspond to clopen subsets of the base space (see 
Sect. 3). This exposes a rich approach to causality [15,33], and opens the possibility of handling relativistic quantum information theory categorically. See also [21], which additionally characterises open subsets of the base space in purely categorical terms. This also invites questions about contextuality $[1,3]$, that might now be addressed within categorical quantum mechanics using regular logic [35].

- Letting the base space vary gives a bicategory of Hilbert bimodules, which forms an infinite continuous extension of the finite higher-categorical approach to categorical quantum mechanics [55] (see Appendix A).

We pay particular attention to Frobenius structures (see Sect. 6), which model classical information flow and algebras of observables [36].

- We prove that dagger Frobenius structures correspond to finite-dimensional $\mathrm{C}^{*}$ algebras that vary continuously over the base space (in Sect. 7). The base space may be considered as modelling spacetime. Thus spacetime protocols can still be modelled within the setting of categorical quantum mechanics [21], and alternative models [11] are not needed.

In fact, we show that this correspondence of objects extends to both $*$-homomorphisms and completely positive maps as morphisms. In other words, we identify the result of applying the $\mathrm{CP}^{*}$-construction [14] to the category of Hilbert modules.

- We reduce studying Frobenius structures to studying commutative ones and central ones (in Sect. 9), and give nontrivial examples of each (in Sect. 6). In fact, commutative Frobenius structures are equivalent to finite coverings of the base space (see Sect. 8). The proof of this fact uses that Frobenius structures have dual objects, otherwise finite branched coverings might also be allowed [43]; we leave open a characterisation of commutative $\mathrm{H}^{*}$-algebras [2]. At any rate, Frobenius structures in a category like that of Hilbert modules need not copy classical information elementwise as previously thought: there may be no copyable states at all. This more intricate structure should inform notions of classicality [33]. On the other hand, classifying central Frobenius structures might be done using a Brauer group [7,45], which we leave to future work.

- The category of Hilbert modules category captures infinite dimension, with entirely standard methods [28], and without dropping unitality [2]: although dagger Frobenius structures form local algebras of observables that are finite-dimensional, globally they can form arbitrary homogeneous $\mathrm{C}^{*}$-algebras [8, IV.1.6].

The article is rounded out by auxiliary results that might be expected: Hilbert modules form a symmetric monoidal dagger category with finite dagger biproducts (see Sect. 2), and the dagger dual objects are precisely the Hilbert modules that are finitely presented projective (see Sect. 5). Finally, we prove (in Sect. 10) that the category of Hilbert modules has dagger kernels only if the base space is totally disconnected, with a view to characterising categories of Hilbert modules. We build on results about Hilbert modules that are fragmented in the literature, but extend them to locally compact spaces, morphisms, and daggers. To keep proofs understandable, we aim for a self-contained account.

\section{Tensor Products of Hilbert Modules}

We start by recalling the basic definitions of Hilbert modules and their morphisms, which form our category of interest. Intuitively, a Hilbert module is a Hilbert space where the base field has been replaced with a $\mathrm{C}^{*}$-algebra. In this article $\mathrm{C}^{*}$-algebras are not necessarily unital. For more information we refer to [40]. 
Definition 2.1. Let $A$ be a $C^{*}$-algebra. A (right) Hilbert $A$-module is a right $A$-module $E$, equipped with a function $\langle-\mid-\rangle_{E}: E \times E \rightarrow A$ that is $A$-linear in the second variable, such that:

$-\langle x \mid y\rangle^{*}=\langle y \mid x\rangle$

$-\langle x \mid x\rangle \geq 0$, and $\langle x \mid x\rangle=0$ if and only if $x=0$;

- $E$ is complete in the norm $\|x\|_{E}^{2}=\|\langle x \mid x\rangle\|_{A}$.

A function $f: E \rightarrow F$ between Hilbert $A$-modules is called bounded by $m \in \mathbb{R}$ when $\|f(x)\|_{F} \leq m\|x\|_{E}$ for all $x \in E$; in this case the infimum of such $m$ is written $\|f\|$. The function $f$ is called adjointable when there exists a function $f^{\dagger}: F \rightarrow E$ satisfying $\langle f(x) \mid y\rangle_{F}=\left\langle x \mid f^{\dagger}(y)\right\rangle_{E}$ for all $x \in E$ and $y \in F$.

Write $\mathbf{H i l b}_{C}^{\mathrm{bd}}$ for the category of Hilbert $C$-modules and bounded $C$-linear functions. A dagger category is a category $\mathbf{C}$ with a functor $\dagger: \mathbb{C}^{\text {op }} \rightarrow \mathbb{C}$ satisfying $X^{\dagger}=X$ on objects and $f^{\dagger \dagger}=f$ on morphisms. Write Hilb $_{C}$ for the dagger category of Hilbert $C$-modules and adjointable functions.

For so-called self-dual Hilbert $A$-modules $E, F$, these two types of morphisms coincide: Hilb bd $_{A}(E, F)=\mathbf{H i l b}_{A}(E, F)[41,3.3-3.4]$.

Our next step is to show that the tensor product of Hilbert modules is well-behaved, in the sense that it makes Hilbert modules into a monoidal dagger category.

If $E$ and $F$ are Hilbert $C$-modules over a commutative $C^{*}$-algebra $C$, another Hilbert $C$-module $E \otimes F$ is given by completing the algebraic tensor product $E \otimes_{\mathbb{C}} F$ with the following inner product and (right) $C$-module structure:

$$
\begin{aligned}
\left\langle x_{1} \otimes y_{1} \mid x_{2} \otimes y_{2}\right\rangle & =\left\langle x_{1} \mid x_{2}\right\rangle\left\langle y_{1} \mid y_{2}\right\rangle, \\
(x \otimes y) c & =x \otimes(y c) .
\end{aligned}
$$

For more details, see Appendix A. A monoidal dagger category is a monoidal category that is also a dagger category in which $(f \otimes g)^{\dagger}=f^{\dagger} \otimes g^{\dagger}$ and the coherence isomorphisms are unitary.

Proposition 2.2. Let $C$ be a commutative $C^{*}$-algebra. The category $\mathbf{H i l b}_{C}^{\mathrm{bd}}$ is symmetric monoidal, and $\mathbf{H i l b}_{C}$ is a symmetric monoidal dagger category.

Proof. If $f: E_{1} \rightarrow E_{2}$ and $g: F_{1} \rightarrow F_{2}$ are bounded maps between Hilbert $C$-modules, we may define $f \otimes g: E_{1} \otimes F_{1} \rightarrow E_{2} \otimes F_{2}$ as the continuous linear extension of $x \otimes y \mapsto f(x) \otimes g(y)$. If $f, g$ were adjointable, then $f \otimes g$ is adjointable with adjoint $f^{\dagger} \otimes g^{\dagger}$ :

$$
\begin{aligned}
\left\langle(f \otimes g)\left(x_{1} \otimes y_{1}\right) \mid x_{2} \otimes y_{2}\right\rangle & =\left\langle f\left(x_{1}\right) \mid y_{1}\right\rangle\left\langle g\left(y_{1}\right) \mid y_{2}\right\rangle \\
& =\left\langle x_{1} \mid f^{\dagger}\left(y_{1}\right)\right\rangle\left\langle y_{1} \mid g^{\dagger}\left(y_{2}\right)\right\rangle \\
& =\left\langle x_{1} \otimes x_{2} \mid\left(f^{\dagger} \otimes g^{\dagger}\right)\left(x_{2} \otimes y_{2}\right)\right\rangle .
\end{aligned}
$$

Clearly id $\otimes \mathrm{id}=\mathrm{id}$ and $(f \circ g) \otimes(h \circ k)=(f \otimes h) \circ(g \otimes k)$, making the tensor product into a functor Hilb bd $_{C}^{\text {bd }} \times$ Hilb $_{C}^{\text {bd }} \rightarrow$ Hilb $_{C}^{\text {bd }}$.

There are functions $\lambda_{E}: C \otimes E \rightarrow E, \rho_{E}: E \otimes C \rightarrow E$, and $\alpha_{E, F, G}: E \otimes(F \otimes$ $G) \rightarrow(E \otimes F) \otimes G$, that continuously extend their algebraic counterparts. Thus they satisfy the pentagon and triangle equalities. It is clear that $\alpha_{E, F, G}$ is unitary, but this is not immediate for $\lambda_{E}$ and $\rho_{E}$. Recall the precise description of the tensor product in Appendix A: it involves the $*$-homomorphism $C \rightarrow \mathcal{L}(E)$ that sends $f$ to $x \mapsto x f$. 
This $*$-homomorphism is nondegenerate [40, p. 5]: if $f_{n}$ is an approximate unit for $C$, and $x \in E$, then

$$
\lim _{n}\left\langle x-x f_{n} \mid x-x f_{n}\right\rangle=\lim _{n}\langle x \mid x\rangle-f_{n}\langle x \mid x\rangle-\langle x \mid x\rangle f_{n}+f_{n}\langle x \mid x\rangle f_{n}=0,
$$

so $E C$ is dense in $E$. Now $\lambda_{E}: C \otimes E \rightarrow E$ is defined by $f \otimes x \mapsto x f$. Therefore

$$
\begin{aligned}
\left\|\lambda_{E}\left(\sum f_{i} \otimes x_{i}\right)\right\|_{E}^{2} & =\left\|\sum x_{i} f_{i}\right\|_{E}^{2} \\
& =\left\|\sum\left\langle x_{i} f_{i} \mid x_{j} f_{j}\right\rangle_{E}\right\|_{C} \\
& =\left\|\sum\left\langle x_{i} \mid x_{j}\right\rangle_{E} f_{i}^{*} f_{j}\right\|_{C} \\
& =\left\|\sum\left\langle f_{i} \otimes x_{i} \mid f_{j} \otimes x_{j}\right\rangle_{C \otimes E}\right\|_{C} \\
& =\left\|\sum f_{i} \otimes x_{i}\right\|_{C \otimes E}^{2},
\end{aligned}
$$

so that $\lambda_{E}$ is an isometric surjection $C \otimes E \rightarrow E$, and hence unitary [40, Theorem 3.5]. Similarly, there are unitaries $\sigma_{E, F}: E \otimes F \rightarrow F \otimes E$ satisfying the hexagon equality. Thus Hilb $\mathbf{b}_{C}^{\mathrm{bd}}$ and $\mathbf{H i l b}_{C}$ are symmetric monoidal with unit $C$.

Next, we focus on additive structure in the category of Hilbert modules. A zero object is an object that is initial and terminal at the same time. If a category has a zero object, there is a unique map $0: E \rightarrow F$ that factors through the zero object between any two objects. A category has finite biproducts when it has a zero object and any two objects $E_{1}, E_{2}$ have a product and coproduct $E_{1} \oplus E_{2}$ with projections $p_{n}: E_{1} \oplus E_{2} \rightarrow E_{n}$ and injections $i_{n}: E_{n} \rightarrow E_{1} \oplus E_{2}$ satisfying $p_{n} \circ i_{n}=\mathrm{id}$ and $p_{m} \circ i_{n}=0$ for $m \neq n$. A dagger category has finite dagger biproducts when it has finite biproducts and $i_{n}=p_{n}^{\dagger}$.

Lemma 2.3. The category $\mathbf{H i l b}_{C}^{\mathrm{bd}}$ has finite biproducts; $\mathbf{H i l b}_{C}$ has finite dagger biproducts.

Proof. Clearly the zero-dimensional Hilbert $C$-module $\{0\}$ is simultaneously an initial and terminal object. Binary direct sums [40, p. 5] are well-defined Hilbert $C$-modules. Since the category Vect of vector space has finite biproducts, the universal property is satisfied via the forgetful functor Hilb $\mathbf{b}_{C}^{\mathrm{bd}} \rightarrow$ Vect, and it suffices to show that direct sums are well-defined on morphisms. Clearly, if $f$ and $g$ are bounded, then so is $f \oplus g$. Similarly, $f$ and $g$ are adjointable maps between Hilbert $C$-modules, so is $f \oplus g$ :

$$
\begin{aligned}
\left\langle(f \oplus g)\left(x_{1}, y_{1}\right) \mid\left(x_{2}, y_{2}\right)\right\rangle & =\left\langle f\left(x_{1}\right) \mid x_{2}\right\rangle+\left\langle g\left(y_{1}\right) \mid y_{2}\right\rangle \\
& =\left\langle x_{1} \mid f^{\dagger}\left(x_{2}\right)\right\rangle+\left\langle y_{1} \mid g^{\dagger}\left(y_{2}\right)\right\rangle \\
& =\left\langle\left(x_{1}, y_{1}\right) \mid\left(f^{\dagger} \oplus g^{\dagger}\right)\left(x_{2}, y_{2}\right)\right\rangle .
\end{aligned}
$$

Finally, the injections $E \rightarrow E \oplus F$ given by $x \mapsto(x, 0)$ are clearly adjoint to the projections $E \oplus F \rightarrow E$ given by $(x, y) \mapsto x$.

To conclude this preliminary section, we discuss an important aspect of the theory of Hilbert modules called localization, and show that it, too, behaves well categorically. Can we turn a Hilbert $C$-module into a Hilbert $D$-module? It turns out that such a change of base needs not just a map $D \rightarrow C$ to alter scalar multiplication, but also a map $C \rightarrow D$ to alter inner products. Recall that the multiplier algebra of a $\mathrm{C}^{*}$-algebra $A$ is the unital $\mathrm{C}^{*}$-algebra $M(A)=\mathbf{H i l b}_{A}(A, A)$, that there is an inclusion $\iota: A \hookrightarrow M(A)$, and that 
any completely positive linear map $f: A \rightarrow B$ extends to $M(f): M(A) \rightarrow M(B)$, see [40, p. 15]. A $*$-homomorphism $f: A \rightarrow M(B)$ is nondegenerate when $f(A) B$ is dense in $B$. If $A$ is already unital then $M(A)=A$.

Definition 2.4. A conditional expectation between $C^{*}$-algebras $A \rightarrow B$ consists of a nondegenerate $*$-homomorphism $g: B \longmapsto M(A)$ and a completely positive linear map $f: A \rightarrow B$ satisfying $M(f) \circ g=\iota$. A conditional expectation is strict when $f(a b)=0$ implies $f(a) f(b)=0$ for all positive $a, b \in A$.

See also Appendix B.

Proposition 2.5 (Localization). Let $f: C \rightarrow D$ be a conditional expectation of a unital commutative $C^{*}$-algebra $C$ onto a unital commutative subalgebra $D \subseteq C$. There is a functor $\operatorname{Loc}_{f}: \mathbf{H i l b}_{C}^{\mathrm{bd}} \rightarrow \mathbf{H i l b}_{D}^{\mathrm{bd}}$, that sends an object $E$ to the completion of $E / N_{f}^{E}$, where $E$ is a pre-inner product D-module by $\langle x \mid y\rangle_{D}=f\left(\langle x \mid y\rangle_{C}\right)$, and $N_{f}^{E}=\{x \in$ $\left.E \mid\langle x \mid x\rangle_{D}=0\right\}$. If $f$ is strict then it is (strong) monoidal and restricts to a dagger functor $\operatorname{Loc}_{f}:$ Hilb $_{C} \rightarrow$ Hilb $_{D}$.

The functor $\operatorname{Loc}_{f}$ is called localization [40, p. 57].

Proof. On a morphism $g: E \rightarrow F$, the functor acts as follows. For $x \in E$, notice that $0 \leq|g(x)|^{2} \leq\|f\|^{2}|x|^{2}$ by [40, Proposition 1.2]. Hence $g\left(N_{f}^{E}\right) \subseteq N_{f}^{F}$, making the function $E / N_{f}^{E} \rightarrow F / N_{f}^{F}$ given by $x+N_{f}^{E} \mapsto g(x)+N_{f}^{F}$ well-defined; define its continuous extension to be $\operatorname{Loc}_{f}(g)$.

This clearly respects identity morphisms and composition, making Loc $f$ a welldefined functor. It also preserves daggers when they are available:

$$
\begin{aligned}
\left\langle\operatorname{Loc}_{f}(g)\left(x+N_{f}^{E}\right) \mid y+N_{f}^{F}\right\rangle_{\operatorname{Loc}_{f}(F)} & =f\left(\left\langle g(x)+N_{f}^{F} \mid y+N_{f}^{F}\right\rangle_{F}\right) \\
& =f\left(\left\langle x+n_{f}^{E} \mid g^{\dagger}(y)+N_{f}^{F}\right\rangle_{E}\right) \\
& =\left\langle x+n_{f}^{E} \mid \operatorname{Loc}_{f}\left(g^{\dagger}\right)\left(y+N_{f}^{F}\right)\right\rangle_{\operatorname{Loc}_{f}(E)} .
\end{aligned}
$$

To show that $\operatorname{Loc}_{f}$ is (strong) monoidal, we have to exhibit unitaries $D \rightarrow \operatorname{Loc}_{f}(C)$ and $\operatorname{Loc}_{f}(E) \otimes \operatorname{Loc}_{f}(F) \rightarrow \operatorname{Loc}_{f}(E \otimes F)$. For the latter, take $\left(x+N_{f}^{E}\right) \otimes(y+$ $\left.N_{f}^{F}\right) \mapsto x \otimes y+N_{f}^{E \otimes F}$. This is well-defined because $f$ is strict: if $x+N_{f}^{E}=0$, that is $f\left(\langle x \mid x\rangle_{C}\right)=0$, then $f\left(\langle x \otimes y \mid x \otimes y\rangle_{C}\right)=f\left(\langle x \mid x\rangle_{C}\langle y \mid y\rangle_{C}\right)=f(\langle x|$ $\left.x\rangle_{C}\right) f\left(\langle y \mid y\rangle_{C}\right)=0$ for any $y \in F$, and so $x \otimes y \in N_{f}^{E \otimes F}$. The adjoint of this map is given by $x \otimes y+N_{f}^{E \otimes F} \mapsto\left(x+N_{f}^{E}\right) \otimes\left(y+N_{f}^{F}\right)$ :

$$
\begin{aligned}
& \left\langle\left(x_{1}+N_{f}^{E}\right) \otimes\left(y_{1}+N_{f}^{F}\right) \mid\left(x_{2}+N_{f}^{E}\right) \otimes\left(y_{2}+N_{f}^{F}\right)\right\rangle_{\operatorname{Loc}_{f}(E) \otimes \operatorname{Loc}_{f}(F)} \\
& =f\left(\left\langle x_{1} \mid x_{2}\right\rangle_{E}\right) \cdot f\left(\left\langle y_{1} \mid y_{2}\right\rangle_{F}\right) \\
& =f\left(\left\langle x_{1} \mid x_{2}\right\rangle_{E} \cdot\left\langle y_{1} \mid y_{2}\right\rangle_{F}\right) \\
& =\left\langle x_{1} \otimes y_{1}+N_{f}^{E \otimes F} \mid x_{2} \otimes y_{2}+N_{f}^{E \otimes F}\right\rangle_{\operatorname{Loc}_{f}(E \otimes F) .}
\end{aligned}
$$

This is well-defined again because $f$ is strict: if $x \otimes y \in N_{f}^{E \otimes F}$, that is $f\left(\langle x \mid x\rangle_{C}\langle y|\right.$ $\left.y\rangle_{C}\right)=0$, then also $\left\langle\left(x+N_{f}^{E}\right) \otimes\left(y+N_{f}^{F}\right) \mid\left(x+N_{f}^{E}\right) \otimes\left(y+N_{f}^{F}\right)\right\rangle=f\left(\langle x \mid x\rangle_{C}\right) f(\langle y|$ $\left.y\rangle_{C}\right)=f\left(\langle x \mid x\rangle_{C}\langle y \mid y\rangle_{C}\right)=0$. These maps are clearly each others inverse.

For the unitary map $D \rightarrow \operatorname{Loc}_{f}(C)$, recall that $\operatorname{Loc}_{f}(C)$ is the completion of $C / N_{f}^{C}$ with $\left\langle c \mid c^{\prime}\right\rangle=f\left(c^{*} c^{\prime}\right)$ and $N_{f}^{C}=\left\{c \in C \mid f\left(c^{*} c\right)=0\right\}$. Consider the map $D \rightarrow$ 
$\operatorname{Loc}_{f}(C)$ given by $d \mapsto d+N_{f}^{C}$, and the map $\operatorname{Loc}_{f}(C) \rightarrow D$ given by $c+n_{f}^{C} \mapsto f(c)$. The latter is well-defined as $c-c^{\prime} \in N_{f}^{C}$ implies $f\left(c-c^{\prime}\right)^{*} f\left(c-c^{\prime}\right)=0$ and hence $f(c)=f\left(c^{\prime}\right)$. They are adjoint because $f$ is $D$-linear:

$$
\langle d \mid f(c)\rangle_{D}=d^{*} f(c)=f\left(d^{*} c\right)=\left\langle d \mid c+N_{f}^{C}\right\rangle_{\operatorname{Loc}_{f}(C)} .
$$

Finally, they are inverses: on the one hand $f(d)=d$ for $d \in D$; on the other hand and $c-f(c) \in N_{f}^{C}$ since

$$
\begin{aligned}
f\left((c-f(c))^{*}(c-f(c))\right) & =f\left(c^{*} c\right)-f\left(f(c)^{*} c\right)-f\left(c^{*} f(c)\right)+f\left(f(c)^{*} f(c)\right) \\
& =f\left(c^{*} c\right)-f(c)^{*} f(c)=0
\end{aligned}
$$

by the Schwartz inequality for completely positive maps [42, Exercise 3.4] and [53, Theorem 1]. The required coherence diagrams are easily seen to commute. Thus Loc $f$ is a (strong) monoidal functor.

Remark 2.6. Not every conditional expectation is strict. For example, take $C=\mathbb{C}^{2}$, and regard $D=\mathbb{C}$ as a subalgebra of $C$ via $z \mapsto(z, z)$. Then $f(u, v)=u+v$ defines a conditional expectation $f: C \rightarrow D$. But taking $a=(1,0)$, and $b=(0,1)$ shows that $f(a b)=f(0,0)=0$ but $f(a) f(b)=1 \cdot 1=1 \neq 0$. Hence for $E=F=C$, the canonical map $\operatorname{Loc}_{f}(E) \otimes \operatorname{Loc}_{f}(F) \rightarrow \operatorname{Loc}_{f}(E \otimes F)$ is not adjointable, that is, not a morphism Hilb $_{C(X)}$.

We will be using Urysohn's lemma for locally compact spaces often [46, 2.12].

Lemma 2.7 (Urysohn). If $X$ is a locally compact Hausdorff space, and $K \subseteq V \subseteq X$ with $K$ compact and $V$ open, then there exists a continuous function $\varphi: X \rightarrow[0,1]$ that is 1 on $K$ and is 0 outside a compact subset of $V$.

Example 2.8. Any point $t$ in a locally compact Hausdorff space $X$ gives rise to a strict conditional expectation as follows. The completely positive map $f: C_{0}(X) \rightarrow \mathbb{C}$ evaluates at $t$. The $*$-homomorphism $g: \mathbb{C} \rightarrow M\left(C_{0}(X)\right)$ is determined by $g(z)(\varphi)=z \varphi$. This clearly satisfies $M(f) \circ g(z)=z$, and is strict because $f$ is multiplicative. This localization at $t \in X$ is the setting Proposition 2.5 will be applied in below.

Remark 2.9. We will also use the previous lemma in the form of Tietze's extension theorem: if $X$ is a locally compact Hausdorff space, and $K \subseteq X$ compact, then any function in $C(K)$ extends to a function in $C_{0}(X)$.

\section{Scalars}

In this section, we investigate how much of the base space internalizes to the category of Hilbert modules over it. It will turn out that we need to look at morphisms into the tensor unit.

Can we get more information about $X$ from Hilb $_{C_{0}(X)}$ by purely categorical means? We first investigate scalars: endomorphisms $I \rightarrow I$ of the tensor unit in a monoidal category. They form a commutative monoid. In the presence of biproducts, they form a semiring, and in the presence of a dagger, they pick up an involution [36].

Lemma 3.1. If $X$ is a locally compact Hausdorff space, there is a *-isomorphism between scalars of $\mathbf{H i l b}_{C_{0}(X)}$ and $C_{b}(X)$, the bounded continuous complex-valued functions on $X$. The same holds for $\mathbf{H i l b}_{C_{0}(X)}^{\mathrm{bd}}$. 
Proof. Recall that a closed ideal $I \subseteq A$ of a $C^{*}$-algebra is essential when $a I=\{0\}$ implies $a=0$ for all $a \in A$. We claim that $C_{0}(X)$ is an essential ideal of the $\mathrm{C}^{*_{-}}$ algebra $\mathcal{L}\left(C_{0}(X)\right)$ of scalars of $\mathbf{H i l b}_{C_{0}(X)}$. Seeing that $C_{0}(X)$ is an ideal in $\mathcal{L}\left(C_{0}(X)\right)$ comes down to showing that for each $f \in C_{0}(X)$ and scalar $s \in \mathcal{L}\left(C_{0}(X)\right)$, there exists $g \in C_{0}(X)$ such that for all $h \in C_{0}(X)$ we have $h g=s(h) f$; choose $g=s(f)$. Seeing that the ideal is essential comes down to showing that for each scalar $s \in \mathcal{L}\left(C_{0}(X)\right)$, if $s(f) g=0$ for all $f, g \in C_{0}(X)$, then $s=0$; given $f \in C_{0}(X)$, choosing $g=s(f)^{*}$ shows that $s(f)^{*} s(f)=0$ implies $\|s(f)\|^{2}=0$ and hence $s(f)=0$. It follows that the scalars of $\mathbf{H i l b}_{C_{0}(X)}$ are precisely the multiplier algebra of $C_{0}(X)$, which is $C_{b}(X)$, see [40, pp. 14-15].

It follows that for compact $X$, the scalars in $\mathbf{H i l b}_{C(X)}$ simply form $C(X)$ itself: any $f \in C(X)$ gives a scalar by multiplication, and all scalars arise that way.

Remark 3.2. If $A$ is a noncommutative $\mathrm{C}^{*}$-algebra, then $\mathbf{H i l b}_{A}$ is a perfectly well-defined dagger category. However, it cannot be monoidal with $A$ as monoidal unit. That is, Proposition 2.2 does not generalise to noncommutative $A$. After all, there is an injective monoid homomorphism $A \hookrightarrow \operatorname{Hilb}_{A}(A, A)$ that sends $a$ to $b \mapsto b a$, which contradicts commutativity of the latter monoid [39, Proposition 6.1].

Next we investigate subobjects. A (dagger) subobject of $E$ is a monomorphism $u: U \rightarrow E$ (satisfying $u^{\dagger} \circ u=\mathrm{id}$ ) considered up to isomorphism of $U$.

Lemma 3.3. There is an isomorphism of partially ordered sets between clopen subsets of a locally compact Hausdorff space $X$ and (dagger) subobjects of the tensor unit $C_{0}(X)$ in Hilb $_{C_{0}(X)}$.

Proof. We will first establish a bijection between clopen subsets of $X$ and subobjects $E \longmapsto C_{0}(X)$ such that $C_{0}(X)=E \oplus E^{\perp}$.

Given a clopen subset $U \subseteq X$, take $E=\left\{f \in C_{0}(X) \mid f(U)=0\right\}$. This is a well-defined Hilbert $C_{0}(X)$-module under the inherited inner product $\langle f \mid g\rangle=f^{*} g$. Then $E^{\perp}=\left\{f \in C_{0}(X) \mid f(X \backslash U)=0\right\}$, and indeed $C_{0}(X)=E \oplus E^{\perp}$.

Conversely, the image of a complemented subobject $E \longmapsto C_{0}(X)$ is a closed ideal of $C_{0}(X)$, and hence is of the form $E=\left\{f \in C_{0}(X) \mid f(U)=0\right\}$ for a closed subset $U \subseteq X$. Because the same holds for $E^{\perp}$ and $C_{0}(X)=E \oplus E^{\perp}$, the closed subset $U$ must in fact be clopen. Taking into account that subobjects are defined up to isomorphism, these two constructions are each other's inverse.

Finally, we prove that any subobject of $C_{0}(X)$ in $\mathbf{H i l b}_{C_{0}(X)}$ is complemented, so that every subobject is a dagger subobject by Lemma 2.3. See also [25, Theorem 3.1]. If $U \subseteq X$ is arbitrary, $E=\left\{f \in C_{0}(X) \mid f(U)=0\right\}=\{f \mid f(\bar{U})=0\}$ is a well-defined object in Hilb $_{C_{0}(X)}$, but the inclusion $i: E \hookrightarrow C_{0}(X)$ is not necessarily a well-defined morphism. Suppose $i$ were adjointable, so that $f(t)^{*} g(t)=f(t)^{*} i^{\dagger}(g)(t)$ for all $t \in X$ and $f, g \in C_{0}(X)$ with $f(U)=0$. If $t \notin \bar{U}$, Urysohn's lemma provides a continuous function $f: X \rightarrow[0,1]$ such that $f(\bar{U})=0$ and $f(t)=1$. Hence $i^{\dagger}(g)(t)=g(t)$ for $t \in X \backslash \bar{U}$. But to make $i^{\dagger}$ well-defined, $i^{\dagger}(g)(t)=0$ for $t \in \bar{U}$, and $i^{\dagger}(g)$ must be continuous. Letting $g$ range over an approximate unit for $C_{0}(X)$ shows that $\bar{U}$ must be clopen.

It follows that there is a bijection between the clopen subsets of a locally compact Hausdorff space $X$ and self-adjoint idempotent scalars in Hilb $_{C_{0}(X)}$ : a dagger subobject $f: E \longmapsto C_{0}(X)$ induces the scalar $s=f \circ f^{\dagger}$, and conversely, the image of a self-adjoint idempotent scalar $s: C_{0}(X) \rightarrow C_{0}(X)$ is a C*-subalgebra $f: E \longmapsto C_{0}(X)$. 
Lemma 3.4. The monoidal categories $\mathbf{H i l b}_{C_{0}(X)}$ and $\mathbf{H i l b}_{C_{0}(X)}^{\mathrm{bd}}$ are monoidally wellpointed: if $f, g: E_{1} \otimes E_{2} \rightarrow F_{1} \otimes F_{2}$ satisfy $f \circ(x \otimes y)=g \circ(x \otimes y)$ for all morphisms $x: C_{0}(X) \rightarrow E_{1}$ and $y: C_{0}(X) \rightarrow E_{2}$, then $f=g$.

Proof. Any element $x \in E$ gives rise to a morphism $C_{0}(X) \rightarrow E$ given by $\varphi \mapsto x \varphi$ with adjoint $\langle x \mid-\rangle_{E}$.

\section{Hilbert Bundles}

Hilbert modules are principally algebraic structures. This section discusses a geometric description, in terms of bundles of Hilbert spaces. While most of this material is wellknown [18], we state it in a way that is useful for our purposes. We will use the following definition of vector bundle in a Hilbert setting.

Definition 4.1. A Hilbert bundle is a bundle $p: E \rightarrow X$ such that:

(a) all fibres $E_{t}$ for $t \in X$ are Hilbert spaces;

(b) any $t_{0} \in X$ has an open neighbourhood $U \subseteq X$, a natural number $n$, and sections $s_{1}, \ldots, s_{n}: U \rightarrow E$ such that:

(i) $\left\{s_{1}(t), \ldots, s_{n}(t)\right\}$ is an orthonormal basis of $E_{t}$ for each $t \in U$;

(ii) the map $(t, \lambda) \mapsto \sum \lambda_{i} s_{i}(t)$ is a homeomorphism $U \times \mathbb{C}^{n} \simeq E_{U}$.

The dimension of the Hilbert bundle is the function that assigns to each $t \in X$ the cardinal number $\operatorname{dim}\left(E_{t}\right)$. The Hilbert bundle is finite when its dimension function is bounded: $\sup _{t \in X} \operatorname{dim}\left(E_{t}\right)<\infty$.

Notice that a Hilbert bundle is a vector bundle. Notice also that any Hilbert bundle over a compact space $X$ is necessarily finite: because $X$ is covered by the open neighbourhoods of each $t_{0} \in X$ given by (b), there is a finite subcover, and the supremum of $\operatorname{dim}\left(E_{t}\right)$ is a maximum ranging over that finite index set and is therefore always finite.

Remark 4.2. It follows from Definition 4.1(b) for a finite Hilbert bundle, the dimension $t \mapsto \operatorname{dim}\left(E_{t}\right)$ is a continuous function $X \mapsto \mathbb{N}$.

Definition 4.1 is a simplification of a few variations in the literature, that we now compare. The reader that is only interested in new developments can safely skip this and continue reading at Definition 4.6. The $\varepsilon$-tube around a local section $s$ of a bundle $p: E \rightarrow X$ whose fibres are normed vector spaces is defined as

$$
T_{\varepsilon}(s)=\left\{x \in E \mid \forall t \in U:\|x-s(p(x))\|_{E_{t}}<\varepsilon\right\} .
$$

A bounded section $s$ is a section whose norm $\|s\|=\sup _{t \in X}\|s(t)\|$ is bounded.

Definition 4.3. A field of Banach (Hilbert) spaces is a bundle $p: E \rightarrow X$ with:

1. all fibres $E_{t}$ for $t \in X$ are Banach (Hilbert) spaces;

2. addition is a continuous function $\left\{(x, y) \in E^{2} \mid p(x)=p(y)\right\} \rightarrow E$;

3. scalar multiplication is a continuous function $\mathbb{C} \times E \rightarrow E$;

4. the norm is a continuous function $E \rightarrow \mathbb{C}$;

5. each $x_{0} \in E$ has a local section $s$ with $s\left(p\left(x_{0}\right)\right)=x_{0}$, and $x_{0}$ has a neighbourhood basis $T_{\varepsilon}(s) \cap E_{U}$ for some neighbourhood $U \subseteq X$ of $p\left(x_{0}\right)$.

We say $p$ has locally finite rank when: 
6. any $t_{0} \in X$ has a neighbourhood $U \subseteq X$ and $n \in \mathbb{N}$ such that $\operatorname{dim}\left(E_{t}\right)=n$ for all $t \in U$.

Finally, a field of Hilbert spaces is finite when the dimension of its fibres is bounded.

Remark 4.4. Definition 4.3 occurs in various places in the literature:

- [20, Definition 2.1]: using the polarization identity we may replace (4) with inner product being a continuous function $\left\{(x, y) \in E^{2} \mid p(x)=p(y)\right\} \rightarrow \mathbb{C}$.

- [19, Definition 1] and [8, IV.1.6.11] replace (5) with the existence of a set $\Delta \subseteq$ $\prod_{t \in X} E_{t}$ satisfying:

- $\{s(t) \mid s \in \Delta\} \subseteq E_{t}$ is dense for all $t \in X$;

- for every $s, s^{\prime} \in \Delta$ the map $x \mapsto\left\langle s(x) \mid s^{\prime}(x)\right\rangle_{E_{t}}$ is in $C(X)$;

- $\Delta$ is locally uniformly closed: if $s \in \prod_{t \in X} E_{t}$ and for each $\varepsilon>0$ and each $t \in X$, there is an $s^{\prime} \in \Delta$ such that $\left\|s\left(t^{\prime}\right)-s^{\prime}\left(t^{\prime}\right)\right\|<\varepsilon$ on a neighbourhood of $t$, then $s \in \Delta$;

this is equivalent because we can recover $E$ as $\prod_{t \in X} E_{t}$ with the topology generated by the basic open sets $T_{\varepsilon}(s) \cap E_{U}$ for $\varepsilon>0$, and $U \subseteq X$ open, and $s \in \Delta$; this topology makes $\Delta$ into the set of bounded sections;

- [20, Definition 2.1] explicitly takes $p$ to be open, which follows from (5), because it also considers a weaker version of (5);

- $[49$, Definition 3.4] takes $s$ in (5) to be a global section, because it also considers spaces $X$ that are not functionally separated; for locally compact Hausdorff spaces $X$ this is equivalent;

- finite fields of Hilbert spaces are usually called uniformly finite-dimensional, and automatically have locally finite rank.

None of these variations matter for the material below.

Lemma 4.5. A Hilbert bundle is the same thing as a field of Hilbert spaces of locally finite rank. A finite Hilbert bundle is the same thing as a finite field of Hilbert spaces.

Proof. First assume that $p: E \rightarrow X$ is a field of Hilbert spaces of locally finite rank. Condition (a) of Definition 4.1 is precisely condition (1) of Definition 4.3. For condition (b), let $t_{0} \in X$. Then (6) yields $n \in \mathbb{N}$ with $\operatorname{dim}\left(E_{t_{0}}\right)=n$. Pick an orthonormal basis $x_{1}, \ldots, x_{n} \in E_{t_{0}}$. Then (5) gives continuous sections $s_{1}^{\prime}, \ldots, s_{n}^{\prime}$ of $p$ over $U_{1}, \ldots, U_{n} \subseteq$ $X$. Take $U=U_{1} \cap \cdots \cap U_{n} \cap\left\{t \in X \mid\left\{s_{1}(t), \ldots, s_{n}(t)\right\}\right.$ linearly independent $\}$; this is an open subset of $X$ by (6) and [20, Proposition 1.6]. Now, as in [20, Proposition 2.3], applying Gram-Schmidt for each $t \in U$ gives continuous sections $s_{1}, \ldots, s_{n}$ of $p$ over $U$ because of (2), (3) and (4). Moreover, these sections $s_{i}$ satisfy (i), (ii), and (iii) of condition (b).

Now assume $p: E \rightarrow X$ is a Hilbert bundle. Condition (1) is still precisely condition (a). For condition (2), define addition $\bigsqcup_{t_{0} \in X} E_{t_{0}}^{2} \rightarrow E_{t_{0}} \subseteq E$ as the cotuple of the additions $E_{t_{0}}^{2} \rightarrow E_{t_{0}}$ over all $t_{0} \in X$. Since the forgetful functor Top $\rightarrow$ Set uniquely lifts colimits, the former is continuous because the latter are continuous by (a). For condition (3), define scalar multiplication $\mathbb{C} \times E \simeq \mathbb{C} \times \bigsqcup_{t_{0} \in X} E_{t_{0}} \simeq \bigsqcup_{t_{0} \in X} \mathbb{C} \times$ $E_{t_{0}} \rightarrow E$ as the cotuple of scalar multiplications $\mathbb{C} \times E_{t_{0}} \rightarrow E_{t_{0}}$ over all $t_{0} \in X$. Again, this is continuous by condition (a). Condition (4) is satisfied exactly like (2). For condition (5), let $x_{0} \in E$. Condition (b) gives a neighbourhood $U \subseteq X$ of $t_{0}=p\left(x_{0}\right)$ and $s_{1}, \ldots, s_{n}: U \rightarrow E$. Define $s: U \rightarrow E_{U} \subseteq E$ by $s(t)=\sum_{i} \lambda_{i} s_{i}(t)$. Then $s\left(p\left(x_{0}\right)\right)=x_{0}$ by (b.ii), and $s$ is continuous on $U$. Let $V \subseteq E$ be a neighbourhood of $x_{0}$. Find a neighbourhood $U_{0} \subseteq X$ of $t_{0}$ with $p(V) \subseteq U_{0}$. Write $\varphi$ for the homeomorphism 
of (b.ii). Take $\varepsilon=1$, and $V_{0}=\varphi\left(U_{0} \times \mathbb{C}^{n}\right)$. Then $x_{0} \in V_{0} \subseteq V$ by construction, and moreover $V_{0}$ is contained in

$$
\begin{aligned}
T_{\varepsilon}(s) \cap E_{U} & =\left\{x \in E_{U} \mid \forall t \in U:\|x-s(p(x))\|_{E_{t}}<1\right\} \\
& =\varphi\left(\left\{\left(t, \lambda_{1}, \ldots, \lambda_{n}\right) \in U \times \mathbb{C}^{n} \mid\left\|\sum_{i=1}^{n} \lambda_{i} s_{i}(t)-s\left(p\left(\sum_{i=1}^{n} \lambda_{i} s_{i}(t)\right)\right)\right\|<1\right\}\right) \\
& =\varphi\left(U \times \mathbb{C}^{n}\right)
\end{aligned}
$$

because $s(t) \in E_{t}$ by (b.ii) and hence $p(s(t))=t$ by (b.i). Finally, condition (6) follows directly from (b).

Having defined the notion of Hilbert bundle of use to us, we now define the appropriate notion of morphisms.

Definition 4.6. A bundle map from $p: E \rightarrow X$ to $p^{\prime}: E^{\prime} \rightarrow X$ is a continuous function $f: E \rightarrow E^{\prime}$ satisfying $p^{\prime} \circ f=p$. Write FieldHilb ${ }_{X}^{\mathrm{bd}}$ for the category of fields of Hilbert spaces and fibrewise linear bundle maps, HilbBundle ${ }_{X}^{\text {bd }}$ for the full subcategory of Hilbert bundles, and FHilbBundle ${ }_{X}^{\text {bd }}$ for the full subcategory of finite Hilbert bundles.

A bundle map $f: p \rightarrow p^{\prime}$ between fields of Hilbert spaces is adjointable when it is adjointable on each fibre, and the map $E_{t}^{\prime} \ni y \mapsto f^{\dagger}(y) \in E_{t}$ is continuous. Write FieldHilb $_{X}$, HilbBundle ${ }_{X}$, and FHilbBundle ${ }_{X}$ for the wide dagger subcategories of adjointable maps.

In the rest of this section we show that it is completely equivalent to work in terms of Hilbert modules, and to work in terms of Hilbert bundles. More precisely, there is a version of the Serre-Swan theorem [56, 13.4.5] for Hilbert bundles, that we now embark on proving. We first establish a functor, then prove that it is an equivalence, and finally that they preserve monoidal structure. If $p: E \rightarrow X$ is a field of Hilbert spaces, we say a function $s: X \rightarrow E$ vanishes at infinity when for each $\varepsilon>0$ there is a compact $U \subseteq X$ such that $\|s(t)\|_{E_{t}}<\varepsilon$ for $t \in X \backslash U$.

Proposition 4.7. Let $X$ be a locally compact Hausdorff space. There is a functor $\Gamma_{0}$ : FieldHilb $_{X}^{\text {bd }} \rightarrow$ Hilb $_{C_{0}(X)}^{\text {bd }}$, defined by

$$
\begin{aligned}
& \Gamma_{0}(p)=\left\{s: X \rightarrow E \mid p \circ s=1_{X}, s \text { continuous, } s \text { vanishes at infinity }\right\} \\
& \Gamma_{0}(f)=f \circ(-) .
\end{aligned}
$$

It restricts to a functor $\Gamma_{0}$ : FieldHilb $\mathbf{b}_{X} \rightarrow \mathbf{H i l b}_{C_{0}(X)}$ that preserves daggers.

Proof. Pointwise multiplication makes $\Gamma_{0}(p)$ into a right $C_{0}(X)$-module. For $s, s^{\prime} \in$ $\Gamma_{0}(p)$ and $t \in X$, the nondegenerate inner product $\left\langle s \mid s^{\prime}\right\rangle(t)=\left\langle s(t) \mid s^{\prime}(t)\right\rangle_{E_{t}}$ takes values in $C_{0}(X)$ by the Cauchy-Schwarz inequality. Finally, $\Gamma_{0}(p)$ is complete: if $s_{n}$ is a Cauchy sequence in $\Gamma_{0}(p)$, then $s_{n}(t)$ is a Cauchy sequence in $E_{t}$ for each $t \in X$, and hence converges to some $s(t)$; since the convergence is uniform this defines a continuous function $s: X \rightarrow E$, that satisfies $p \circ s=1_{X}$ and vanishes at infinity by construction. Thus $\Gamma_{0}(p)$ is a well-defined Hilbert $C_{0}(X)$-module.

Let $f: p \rightarrow p^{\prime}$ be a morphism of fields of Hilbert spaces. Define $\Gamma_{0}(f)=f \circ$ $(-): \Gamma_{0}(p) \rightarrow \Gamma_{0}\left(p^{\prime}\right)$. This is clearly $C_{0}(X)$-linear, bounded, and functorial. It is also well-defined: if $s \in \Gamma_{0}(p)$, then $\|f \circ s\| \leq\|f\|\|s\|$ vanishes at infinity too. 
A morphism $f: p \rightarrow p^{\prime}$ in FieldHilb F $_{X}$ is adjointable precisely when there is a bounded bundle map $f^{\dagger}: p^{\prime} \rightarrow p$ that provides fibrewise adjoints:

$$
\left\langle f(s(t)) \mid s^{\prime}(t)\right\rangle_{E_{t}}=\left\langle s(t) \mid f^{\dagger}\left(s^{\prime}(t)\right)\right\rangle_{E_{t}^{\prime}}
$$

for all $t \in X, s \in \Gamma_{0}(p)$, and $s^{\prime} \in \Gamma_{0}\left(p^{\prime}\right)$. That is, $f$ is adjointable if and only if $\Gamma(f)$ is. Thus the functor $\Gamma_{0}$ preserves daggers.

\section{Theorem 4.8. The functors $\Gamma_{0}$ from Proposition 4.7 are equivalences.}

Proof. We first show that the functor $\Gamma_{0}$ is faithful. Suppose $f \neq g$, say $f(x) \neq g(x)$ and $p(x)=t$. There exists a local continuous section $s_{U}: U \rightarrow E$ of $p$ over some open neighbourhood $U \subseteq X$ because $p$ is a field of Hilbert spaces. Local compactness of $X$ ensures there is a compact neighbourhood of $t$ within $U$, which in turn contains an open neighbourhood $V \subseteq X$ of $x$. Urysohn's lemma provides a continuous function $r: X \rightarrow[0,1]$ that vanishes on $X \backslash V$ and satisfies $r(t)=1$. Now define $s_{X}: X \rightarrow E$ by $s_{x}(t)=0$ for $t \in X \backslash U$ and $s_{X}(t)=r(t) s_{U}(t)$ for $t \in U$. Then $s_{X} \in \Gamma_{0}(p)$ and $s_{x}(t)=x$. Hence $f \circ s_{x}(t) \neq g \circ s_{x}(t)$, and so $\Gamma_{0}(f) \neq \Gamma_{0}(g)$.

Next we show that the functor $\Gamma_{0}$ is also full. Suppose $f: \Gamma_{0}(p) \rightarrow \Gamma_{0}\left(p^{\prime}\right)$ is bounded and $C_{0}(X)$-linear. For $x \in E$, set $g(x)=f\left(s_{x}\right)(p(x))$. Because $s_{x} \in \Gamma_{0}(p)$, now $f\left(s_{x}\right) \in \Gamma_{0}\left(p^{\prime}\right)$, so the value $g(x)=f\left(s_{x}\right)(p(x))$ is an element of $E^{\prime}$. Thus $g: E \rightarrow E^{\prime}$ is a well-defined function, that furthermore satisfies $p^{\prime} \circ g=p$. It is also fibrewise linear because if $p(x)=p(y)$ then $f\left(s_{x}+s_{y}\right)(p(x))=f\left(s_{x+y}\right)(p(y))$. Moreover $g$ is continuous by the definition of the topology on the field of Hilbert spaces $E$. Hence $g$ is a well-defined morphism of fields of Hilbert spaces. Finally, if $s \in \Gamma_{0}(p)$ and $t \in X$, then $g(s(t))=f\left(s_{s(t)}\right)(p(s(t)))=f\left(s_{s(t)}\right)(t)=f(s)(t)$. So $f(s)=g \circ s$, whence $f=\Gamma_{0}(g)$, and $\Gamma_{0}$ is full.

Finally, we show that $\Gamma_{0}$ is essentially surjective. Let $H$ be a $C_{0}(X)$-Hilbert module. Set $E=\bigsqcup_{t \in X} \operatorname{Loc}_{t}(H)$, and let $p$ be the canonical projection $E \rightarrow X$. Because $X$ is locally compact Hausdorff, it is compactly generated: a subset $U \subseteq X$ is open if and only if $U \cap K$ is open in $K$ for all compact subsets $K \subseteq X$. Hence the topology on $X$ is determined by the topology of its compact subspaces. It follows from [16, II.1.15] and [49, Lemma 3.01(iv), Lemma 3.09, and Proposition 3.10] that there is a unique weakest topology on $E$ making $p$ into a field of Hilbert spaces.

As in Lemma 3.4, we may regard elements of $H$ as adjointable maps $C_{0}(X) \rightarrow H$. For $x \in H$, define $s_{x}: X \rightarrow E$ by $s_{x}(t)=\operatorname{Loc}_{t}(x)$, so that $p \circ s_{X}=1_{X}$ by construction. Moreover, $s_{x}$ vanishes at infinity, because the inner product in $H$ takes values in $C_{0}(X)$ : if $\varepsilon>0$, there is a compact $U \subseteq X$ such that $\left\|s_{x}(t)\right\|_{\operatorname{Loc}_{t}(H)}=\|x\|_{H}(t)<\varepsilon$ for $t \in X \backslash U$. Finally, $s_{x}$ is continuous by construction of the topology on $E$. Thus $\left\{s_{x} \mid x \in\right.$ $X\} \subseteq \Gamma_{0}(p)$.

To complete the proof that $\Gamma_{0}$ is essentially surjective, it now suffices to show that $\left\{s_{x} \mid x \in X\right\} \subseteq \Gamma_{0}(p)$ is dense. Let $s \in \Gamma_{0}(p)$ and $\varepsilon$. Then there exists a compact subset $K \subseteq X$ such that $\|s(t)\|<\varepsilon$ for $t \in X \backslash K$. Urysohn's lemma provides a function $X \rightarrow[0,1]$ that vanishes at infinity such that $f(t)=1$ for $t \in K$. By multiplying with this function it suffices to find $x \in H$ so that the continuous local section $s_{x}: K \rightarrow X$ satisfies $\left\|s_{X}(t)-s(t)\right\|<\varepsilon$ for $t \in K$. This can be done by the method of the proof of [49, Theorem 3.12]. Therefore $\left\|s_{x}(t)-s(t)\right\|<\varepsilon$ for all $t \in X$. Thus $\Gamma_{0}(p) \simeq H$, and $\Gamma_{0}$ is essentially surjective.

Corollary 4.9. The category FieldHilb ${ }_{X}^{\text {bd }}$ is a symmetric monoidal category for any topological space $X$, where the tensor product of $E \rightarrow X$ and $F \rightarrow X$ is $E \otimes F=$ 
$\bigsqcup_{t \in X} E_{t} \otimes F_{t}$ (with canonical topology provided by [16, II.1.15] as in the proof of the previous lemma.) The category FieldHilb $_{X}$ is a symmetric monoidal dagger subcategory. The functors $\Gamma_{0}$ are (strong) monoidal.

Proof. The tensor product $E \otimes E^{\prime}$ becomes a well-defined object by letting $\Delta_{E \otimes F}$ be the closure of the pre-Hilbert $C_{0}(X)$-module of all finite sums of bounded sections vanishing at infinity $\sum_{i=1}^{n} s_{i} \otimes s_{i}^{\prime}$ of $s_{i} \in \Gamma_{0}(E)$ and $s_{i}^{\prime} \in \Gamma_{0}\left(E^{\prime}\right)$; see [19, Section 18] or [12, Definition 15.3]. Via Lemma 4.5, this restricts to the monoidal product on FHilbBundle ${ }_{X}$ as in the statement. Defining tensor products of morphisms is straightforward, as are associators and unitors, and checking the pentagon and triangle equations. The dagger is also clearly well-defined in FHilbBundle ${ }_{X}$, making it a symmetric monoidal dagger category. By construction of Proposition 2.2, the functors $\Gamma_{0}$ are (strong) monoidal.

\section{Dual Objects}

After having given the equivalent geometric description of Hilbert modules in terms of bundles in the last section, we now return to studying the monoidal structure. This section is devoted to dual objects, that play an important role in any monoidal category. Dual objects generally behave somewhat like 'finite' or 'finite-dimensional' objects. The precise notion of 'finiteness' in this setting turns out to be that in the following definition.

From now on we will restrict ourselves to locally compact Hausdorff spaces $X$ that are paracompact.

Definition 5.1. A Hilbert $C$-module $E$ is finitely presented projective when there is an adjointable map $i: E \rightarrow C^{n}$ for some $n \in \mathbb{N}$ with $i^{\dagger} \circ i=\mathrm{id}_{E}$.

In other words, finitely presented projective Hilbert $C$-modules are orthogonal direct summands of $C^{n}$. Any (algebraically) finitely generated projective Hilbert $C$-module is an example. When $X$ is compact, a Hilbert $C(X)$-module is finitely presented projective if and only if it is finitely generated as a $C(X)$-module and a projective object in the category of $C(X)$-modules [56, Theorem 5.4.2].

Finitely presented projective Hilbert modules have pleasant properties, such as the following lemma, that proves that all bounded maps are adjointable in this setting.

Lemma 5.2. Any bounded C-linear map between finitely presented projective Hilbert $C$-modules is adjointable.

Proof. Let $i: E \rightarrow C^{m}$ and $j: F \rightarrow C^{n}$ satisfy $i^{\dagger} \circ i=\operatorname{id}_{E}$ and $j^{\dagger} \circ j=\mathrm{id}_{F}$. Let $f: E \rightarrow F$ be a bounded $C$-linear map. Then $g=j \circ f \circ i^{\dagger}: C^{m} \rightarrow C^{n}$ is a bounded $C$-linear map, and hence an $m$-by- $n$ matrix of bounded $C$-linear maps $C \rightarrow C$. But any bounded linear map $C_{0}(X) \rightarrow C_{0}(X)$ is adjointable. To see this, first use Lemma 3.1 to see that it multiplies with some $k \in C_{b}(X)$. Now $\langle k l \mid m\rangle(t)=k(t)^{*} l(t) m(t)=$ $\left\langle l \mid k^{*} m\right\rangle(t)$, so $k$ is adjointable. Thus, by Lemma 2.3, also $g$ is adjointable. But then $f^{\dagger}=i^{\dagger} \circ g^{\dagger} \circ j$ is an adjoint for $f$, because $\left\langle f^{\dagger}(y) \mid x\right\rangle_{E}=\left\langle y \mid j^{\dagger} \circ g \circ i(x)\right\rangle_{F}=$ $\left\langle y \mid j^{\dagger} \circ j \circ f \circ i^{\dagger} \circ i(x)\right\rangle_{F}=\langle y \mid f(x)\rangle_{F}$.

It follows that the full subcategories of $\mathbf{H i l b}_{C}$ and $\mathbf{H i l b}_{C}^{\mathrm{bd}}$ of finitely presented projective Hilbert $C$-modules coincide. We write FHilb $_{C}$ for this category. If $C$ is unital, we write $1_{C}$ for its unit.

There is an established notion of dual Hilbert module, that a priori differs from the categorical notion. The following lemma details the established notion. 
Lemma 5.3. If $X$ is a locally compact Hausdorff space, and $E$ is a finitely presented projective Hilbert $C_{0}(X)$-module, then $E^{*}=\operatorname{Hilb}_{C_{0}(X)}\left(E, C_{0}(X)\right)$ is a Hilbert $C_{0}(X)$ module where $\langle f \mid g\rangle_{E^{*}}$ is the element of $C_{0}(X)$ that $f \circ g^{\dagger}$ multiplies with according to Lemma 3.1. If $X$ is compact, $\langle f \mid g\rangle_{E^{*}}=f \circ g^{\dagger}\left(1_{C(X)}\right)$.

Proof. It is clear that $E^{*}$ is a $C_{0}(X)$-module with pointwise operations. Any $f, g \in E^{*}$ are adjointable by Lemma 5.2, and hence of the form $f=\langle x \mid-\rangle_{E}$ and $g=\langle y \mid-\rangle_{E}$ for $x, y \in E$. Hence $f^{\dagger}(\varphi)=x \varphi$ and $g^{\dagger}(\varphi)=y \varphi$, and $f \circ g^{\dagger}$ is the scalar that multiplies with $\langle x \mid y\rangle_{E} \in C_{0}(X) \subseteq C_{b}(X)$. Hence the inner product $\langle f \mid g\rangle_{E^{*}}=\langle x \mid y\rangle_{E}$ is well-defined. It is clearly sesquilinear and positive semidefinite by Lemma 3.1. It is also nondegenerate: if $\langle f \mid f\rangle_{E^{*}}=0$ for $f=\langle x \mid-\rangle_{E}$, then $\langle x \mid x\rangle=0$, so $x=0$ and hence $f=0$. If $f_{n}$ is a Cauchy sequence in $E^{*}$, say $f_{n}=\left\langle x_{n} \mid-\right\rangle_{E}$, then $x_{n}$ is a Cauchy sequence in $E$ which converges to some $x \in E$, so $f_{n}$ converges to $f=\langle x \mid-\rangle_{E}$ in $E^{*}$.

We call $E^{*}$ the dual Hilbert $C$-module of $E$.

We now move from the concrete to the abstract, and define a categorical notion of dual object.

Definition 5.4. Objects $E, E^{*}$ in a monoidal category are called dual objects when there are morphisms $\zeta: I \rightarrow E^{*} \otimes E$ and $\varepsilon: E \otimes E^{*} \rightarrow I$ making the following diagrams commute:

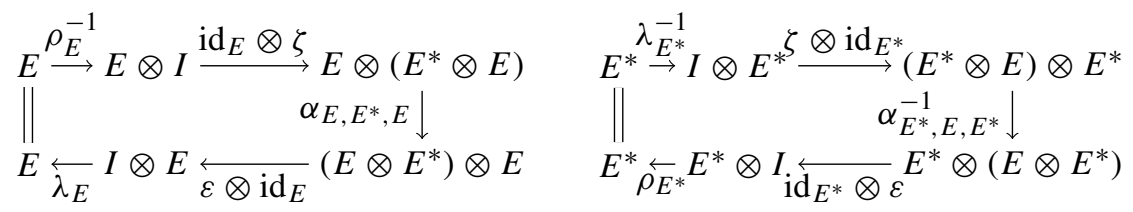

In a symmetric monoidal dagger category, dual objects are dagger dual objects when $\zeta=\sigma \circ \varepsilon^{\dagger}$, where $\sigma: E \otimes E^{*} \rightarrow E^{*} \otimes E$ is the swap map.

If an object has a (dagger) dual, then that dual is unique up to unique (unitary) isomorphism.

A priori, the two notions of dual of a Hilbert module are unrelated. We now show that the categorical notion is equivalent to the concrete notion. In other words, we now show that dual Hilbert $C$-modules are dual objects in the finitely presented projective case over a paracompact space $X$.

Theorem 5.5. Let $X$ be a paracompact locally compact Hausdorff space X. For a Hilbert $C_{0}(X)$-module $E$, the following are equivalent:

(a) $E$ has a dagger dual object in $\mathbf{H i l b}_{C_{0}(X)}$;

(b) $E \simeq \Gamma_{0}(p)$ for a finite Hilbert bundle $p$;

(c) $E$ is finitely presented projective.

Only the implication (b) $\Rightarrow$ (c) requires paracompactness; (a) $\Rightarrow(b)$ and (c) $\Rightarrow$ (a) hold for arbitrary locally compact Hausdorff spaces $X$.

Proof. $\left(\right.$ a) $\Rightarrow\left(\right.$ b): Assume that $E$ has a dagger dual object $E^{*}$. Then also all its localisations $E_{t}=\operatorname{Loc}_{t}(E)$ are dagger dualisable, and so [3, Corollary 19] each $E_{t}$ is a finite-dimensional Hilbert space. Now regard $E$ as a field of Hilbert spaces over $X$ as in Theorem 4.8. Then $\zeta: C_{0}(X) \rightarrow E^{*} \otimes E$ and $\varepsilon: E \otimes E^{*} \rightarrow C_{0}(X)$ are bundle maps 
and hence bounded. But then $\operatorname{dim}\left(E_{t}\right)=\|\varepsilon \circ \sigma \circ \zeta(t)\| \leq\|\varepsilon\|\|\zeta\|$ is bounded, so $E$ is a finite Hilbert bundle.

(b) $\Rightarrow(\mathrm{c})$ : Let $p: E \rightarrow X$ be a finite Hilbert bundle. Then every $t \in X$ has a neighbourhood $U_{t}$ and a homeomorphism $g_{t}: \mathbb{C}^{n_{t}} \times U_{t} \rightarrow p^{-1}\left(U_{t}\right)$ that is fibrewise unitary. This forms a cover $\left\{U_{t}\right\}$ of $X$. Because $X$ is paracompact, we may pick a locally finite refinement $U_{j}$, and a partition of unity $f_{j}: X \rightarrow[0,1]$ subordinate to it: $f_{j}$ vanishes outside $U_{j}$ and $\sum_{j} f_{j}(t)=1$ for all $t \in X$. Because $p$ is finite, the numbers $n_{t}$ are bounded by some $n \in \mathbb{N}$, and the functions $g_{t}$ extend to continuous maps $\mathbb{C}^{n} \times U_{t} \rightarrow p^{-1}\left(U_{t}\right)$ that fibrewise satisfy $g_{t} \circ g_{t}^{\dagger}=\mathrm{id}$. Write $g_{j}$ for the restrictions $\mathbb{C}^{n} \times U_{j} \rightarrow p^{-1}\left(U_{j}\right)$; these are still continuous and fibrewise coisometric. Now $(e, t) \mapsto \sum_{j} g_{j}(e, t) f_{j}(t)$ defines a continuous bundle map $\mathbb{C}^{n} \times X \rightarrow E$ that is (fibrewise) coisometric. Thus $\Gamma_{0}(E)$ is finitely presented projective by Theorem 4.8 .

(c) $\Rightarrow\left(\right.$ a): Assume that $i: E \rightarrow C_{0}(X)^{n}$ satisfies $i^{\dagger} \circ i=\operatorname{id}_{E}$. First, notice that $C_{0}(X)$ is its own dagger dual object, and therefore so is $C_{0}(X)^{n}$. Explicitly,

$$
\zeta: C_{0}(X) \rightarrow\left(\bigoplus_{i=1}^{n} C_{0}(X)\right) \otimes\left(\bigoplus_{j=1}^{n} C_{0}(X)\right) \simeq \bigoplus_{i, j=1}^{n} C_{0}(X)
$$

sends $f \in C_{0}(X)$ to $\left(\delta_{i, j} f\right)_{i, j} \in \bigoplus_{i, j=1}^{n} C_{0}(X)$. Thus (id $\left.\otimes\left(i \circ i^{\dagger}\right)\right) \circ \zeta=\left(\left(i \circ i^{\dagger}\right) \otimes \mathrm{id}\right) \circ \zeta$ because it holds at each $t \in X$ and therefore globally by Theorem 4.8. It follows that the idempotent (id $\otimes \varepsilon) \circ\left(\right.$ id $\otimes\left(i \circ i^{\dagger}\right) \circ$ id $) \otimes(\zeta \otimes$ id $): C_{0}(X)^{n} \rightarrow C_{0}(X)^{n}$ is split by $i: E \rightarrow C_{0}(X)^{n}$. The unit $\left(i^{\dagger} \otimes i^{\dagger}\right) \circ \zeta: C_{0}(X) \rightarrow E \otimes E$ now witnesses that $E$ is a dagger dual object of $E$.

Finally, we show what restricting to dual objects means concretely in terms of bundles, as described in the previous section.

Theorem 5.6. There is a monoidal equivalence of compact (dagger) categories

$$
\text { FHilbBundle }_{X} \stackrel{\Gamma_{0}}{\longrightarrow} \text { FHilb }_{C_{0}(X)}
$$

for any paracompact locally compact Hausdorff space X.

Proof. By Theorem 5.5, the monoidal subcategory FHilb $_{C_{0}(X)}$ of $\mathbf{H i l b}_{C_{0}(X)}$ is compact. Because (strong) monoidal functors preserve dual objects, the inverse image under $\Gamma_{0}$ in FieldHilb bd $_{X}^{\text {bd }}$ is also compact by Corollary 4.9. The dual of $E \rightarrow X$ is given by $\left(E^{*}\right)_{t}=\left(E_{t}\right)^{*}$ (with topology given by [16, II.1.15]). By Lemma 4.5 the functor $\Gamma_{0}$ therefore restricts as in the statement, and is an equivalence by Theorem 4.8 .

It follows that the tensor product of Proposition 2.2 of finitely presented projective Hilbert modules is again finitely presented projective, and so that $\mathbf{F H i l b}_{C_{0}(X)}$ is a symmetric monoidal dagger category. It is also easy to see that $\mathbf{F H i l b}_{C_{0}(X)}$ has finite dagger biproducts.

\section{Frobenius Structures}

We now start the study of dagger Frobenius structures in the category $\mathbf{H i l b}_{C_{0}(X)}$. Many of the results below hold for arbitrary (non-dagger) Frobenius structures, but we focus on dagger Frobenius structures, and leave open the generalisation to purely algebraic proofs. We will occassionally use the graphical calculus, in which dagger becomes horizontal reflection, tensor product becomes drawing side by side, and composition becomes vertical stacking. For more details we refer to [47]. Let's start with the definitions. 
Definition 6.1. A dagger Frobenius structure in $\mathbf{H i l b}_{C}$ is a Hilbert $C$-module $E$ with morphisms $\eta: C \rightarrow E$ and $\mu: E \otimes E \rightarrow E$ satisfying:

$$
\begin{aligned}
\mu \circ(\eta \otimes \mathrm{id}) & =\mathrm{id}=\mu \circ(\mathrm{id} \otimes \eta), \\
\mu \circ(\mu \otimes \mathrm{id}) & =\mu \circ(\mathrm{id} \otimes \mu), \\
(\mu \otimes \mathrm{id}) \circ\left(\mathrm{id} \otimes \mu^{\dagger}\right) & =(\mathrm{id} \otimes \mu) \circ\left(\mu^{\dagger} \otimes \mathrm{id}\right) .
\end{aligned}
$$

or graphically:

$$
a=1=0
$$<smiles>CCCCOCCOC</smiles>

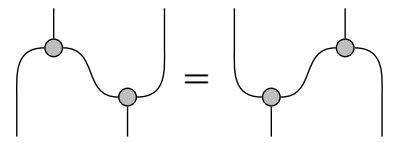

A morphism $d: E \rightarrow E$ is called central when $\mu \circ\left(\operatorname{id}_{E} \otimes d\right)=d \circ \mu=\mu \circ\left(d \otimes \mathrm{id}_{E}\right)$.

$$
C_{\frac{d}{d}}^{d}=\frac{d}{d}=\frac{d}{d}
$$

The dagger Frobenius structure $(E, \mu, \eta)$ is called:

- commutative when $\mu \circ \sigma=\mu$;<smiles>CCOC=C1CC(C)CCO1</smiles>

- special when $\mu \circ \mu^{\dagger}=\mathrm{id}_{E}$;

$$
0_{1}^{1}=
$$

- specialisable when $\mu \circ(d \otimes d) \circ \mu^{\dagger}=\mathrm{id}_{E}$ for a central isomorphism $d=d^{\dagger}$, called the specialiser;

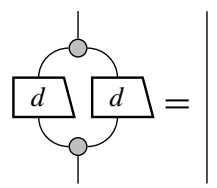

- nondegenerate when $\eta^{\dagger} \circ \eta$ is invertible. 
It follows from the axioms of Frobenius structures that they in fact satisfy the strong Frobenius law:

$$
(\mu \otimes \mathrm{id}) \circ\left(\mathrm{id} \otimes \mu^{\dagger}\right)=\mu^{\dagger} \circ \mu=(\mathrm{id} \otimes \mu) \circ\left(\mu^{\dagger} \otimes \mathrm{id}\right)
$$

or graphically:

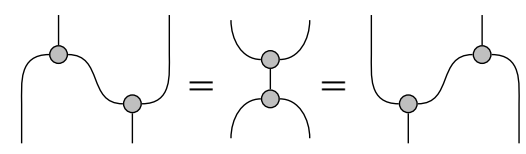

Dagger Frobenius structures are their own dagger dual, with unit $\mu^{\dagger} \circ \eta: I \rightarrow E \otimes E$. Hence dagger Frobenius structures in $\mathbf{H i l b}_{C_{0}(X)}$ live in $\mathbf{F H i l b}_{C_{0}(X)}$ for paracompact $X$. Observe that specialisable dagger Frobenius structures are symmetric; see [14, Proposition 2.7] and notice that our notion of specialisability implies the notion of normalisability used there.

To provide some intuition we now consider the paradigmatic example of a dagger Frobenius structure.

Remark 6.2. For $C=\mathbb{C}$, special dagger Frobenius structures correspond to finitedimensional $C^{*}$-algebras [54]. Any dagger Frobenius structure $E$ in $\mathbf{H i l b}_{C}$ has an involution $i: E \rightarrow E^{*}$ given by (id $\left.\otimes \eta^{\dagger}\right) \circ($ id $\otimes \mu) \circ(\zeta \otimes$ id) $[54,4.4]$. In the graphical calculus, $\mu$ is drawn as $\dot{\alpha}$, and $\eta$ as $\partial$. The involution is thus drawn as follows.

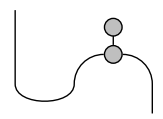

One of our first aims is to generalise this to arbitrary $C$.

Next we define the appropriate morphisms making dagger Frobenius structures with various properties into categories.

Definition 6.3. A *-homomorphism between Frobenius structures in $\mathbf{H i l b}_{C}$ is a morphism $f$ that preserves the involution (2) and the multiplication: $\mu \circ(f \otimes f)=f \circ \mu$, and $f_{*} \circ i=i \circ f$. Write Frob $_{C}$ for the category of specialisable dagger Frobenius structures in $\mathbf{H i l b}_{C}$ with $*$-homomorphisms, and $\mathbf{S F r o b} \mathbf{b}_{C}$ for the full subcategory of special dagger Frobenius structures.

The following proposition shows that, categorically, studying special Frobenius structures teaches us all about specialisable ones.

Proposition 6.4. The categories $\mathbf{F r o b}_{C}$ and $\mathbf{S F r o b}_{C}$ are monoidally equivalent (via the inclusion of the latter into the former).

Proof. Any specialisable dagger Frobenius structure $(E, \mu, \eta)$ is isomorphic to a special one. Namely, let $d$ be the specialiser and define $\mu^{\prime}=d \circ \mu$ and $\eta^{\prime}=d^{-1} \circ \eta$. It is easy to check that $\left(E, \mu^{\prime}, \eta^{\prime}\right)$ is then a special dagger Frobenius structure, and that $d:\left(E, \mu^{\prime}, \eta^{\prime}\right) \rightarrow(E, \mu, \eta)$ and $d^{-1}:(E, \mu, \eta) \rightarrow\left(E, \mu^{\prime}, \eta^{\prime}\right)$ are $*$-homomorphisms that are each other's inverse. See also [34, Lemma 1.2].

The following lemma observes that Frobenius structures behave well under localization, as discussed in Sect. 2. 
Lemma 6.5. If $E$ is a special dagger Frobenius structure in $\mathbf{H i l b}_{C_{0}(X)}$, then all its localizations at $t \in X$ are dagger Frobenius structures in $\mathbf{H i l b}$, and hence finite-dimensional $C^{*}$-algebras.

Proof. Consider the (strong) monoidal dagger functor Hilb $_{C_{0}(X)} \rightarrow$ Hilb of Proposition 2.5 for each $t \in X$. Such functors preserve dagger Frobenius structures, as well as speciality and specialisability.

The following two examples look at one paradigmatic way to construct Frobenius structures in the category of Hilbert modules, generalising Remark 6.2.

Example 6.6. Any finite-dimensional $\mathrm{C}^{*}$-algebra $A$ is a special dagger Frobenius structure in FHilb, and gives rise to a special dagger Frobenius structure $C_{0}(X, A)$ in Hilb $_{C_{0}(X)}$ over a locally compact Hausdorff space $X$. Frobenius structures isomorphic to one of this form are called trivial.

In particular, $\mathbb{M}_{n}\left(C_{0}(X)\right) \simeq C_{0}\left(X, \mathbb{M}_{n}\right)$ is a special dagger Frobenius structures in Hilb $_{C_{0}(X)}$. It follows from Lemma 2.3 that direct sums of such matrix algebras are special dagger Frobenius structures in $\mathbf{H i l b}_{C_{0}(X)}$, too, and up to isomorphism this accounts for all trivial Frobenius structures.

Example 6.7. If $X$ is a paracompact locally compact Hausdorff space, and $E$ is a finitely presented projective Hilbert $C_{0}(X)$-module, then $\mathcal{L}(E)=E^{*} \otimes E$ is a specialisable dagger Frobenius structure.

Proof. This follows from Theorem 5.5 and [14, Proposition 2.11]; take multiplication $\mathrm{id}_{E^{*}} \otimes \varepsilon \otimes \mathrm{id}_{E}: E^{*} \otimes E \otimes E^{*} \otimes E \rightarrow E^{*} \otimes E$ and unit $\eta: C_{0}(X) \rightarrow E^{*} \otimes E$.

Notice that trivial Frobenius structures in $\mathbf{H i l b}_{C_{0}(X)}$ in general need not be direct summands of $C_{0}(X)^{n}$. There are endomorphism algebras that are not direct sums of matrix algebras in Hilb $\mathbf{b}_{C(X)}$. For example, take $X=2$. Then $\mathbb{M}_{n}(\mathbb{C})$ is a corner algebra of $\mathbb{M}_{n}\left(\mathbb{C}^{2}\right)$, but it is not isomorphic to a direct summand of the latter. It is nevertheless the endomorphism algebra of the Hilbert $C(X)$-module $\mathbb{C}^{n}$, but still trivial as a Frobenius structure.

The rest of this section develops nontrivial examples of commutative and central dagger Frobenius structures in $\mathbf{H i l b}_{C_{0}(X)}$. We need some topological preliminaries.

Definition 6.8. A bundle is a continuous surjection $p: Y \rightarrow X$ between topological spaces. Write $Y_{U}=p^{-1}(U)$ for $U \subseteq X$, and $Y_{t}=p^{-1}(t)$ for the fibre over $t \in X$. The bundle is finite when there is a natural number $n$ such that all fibres have cardinality at most $n$. A (local) section over $U$ is a continuous function $s: U \rightarrow Y$ satisfying $p \circ s=\mathrm{id}_{U}$; a global section is a section over $X$. A bundle is a covering when every $t \in X$ has an open neighbourhood $U \subseteq X$ such that $Y_{U}$ is a union of disjoint open sets that are each mapped homeomorphically onto $U$ by $p$.

Example 6.9. Write $S^{1}=\{z \in \mathbb{C}|| z \mid=1\}$ for the unit circle. For any natural number $n$, the map $p: S^{1} \rightarrow S^{1}$ given by $p(z)=z^{n}$ is a finite covering. 


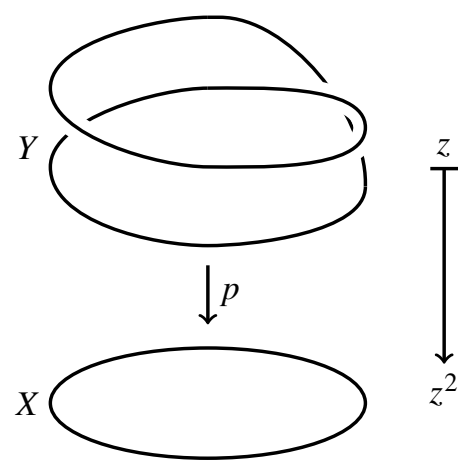

The map $z \mapsto z^{n}$ is also a finite covering on the unit disc $\{z \in \mathbb{C}|| z \mid \leq 1\}$.

Lemma 6.10. If $p: Y \rightarrow X$ is a finite covering between Hausdorff spaces, then the diagonal $\Delta_{Y}=\{(y, y) \mid y \in Y\}$ is a closed and open subset of the pullback $Y \times_{X} Y=$ $\left\{\left(y, y^{\prime}\right) \in Y \times Y \mid p(y)=p\left(y^{\prime}\right)\right\}$ of $p$ along itself.

Proof. Because $Y$ is Hausdorff, the diagonal $\Delta_{Y}$ is closed in $Y \times Y$, and hence also in its closed subspace $Y \times{ }_{X} Y$. To see that $\Delta_{Y}$ is also open, let $y \in Y$, say $p(y)=t$. Then the points $p^{-1}(t)=\left\{y_{1}, \ldots, y_{n}\right\}$ are distinguished by disjoint open neighbourhoods $V_{1}, \ldots, V_{n} \subseteq Y$ that are all mapped homeomorphically onto $U$ by $p$. Say $y=y_{i}$. Set $V=\left(V_{i} \times V_{i}\right) \cap\left(Y \times{ }_{X} Y\right)$. Then $(y, y) \in V$, and $V$ is open in $Y \times{ }_{X} Y$ by definition of the pullback topology. If $v, v^{\prime} \in V_{i}$ satisfy $p(v)=p\left(v^{\prime}\right)$ then $v=v^{\prime}$ because $p: V_{i} \rightarrow U$ is a homeomorphism, so $V$ is contained in $\Delta_{Y}$.

After this topological preparation, we can now construct nontrivial Frobenius structures in the category of Hilbert modules. Later, in Sect. 8, it will turn out that any commutative Frobenius structure arises this way.

Example 6.11. If $p: Y \rightarrow X$ is a covering between locally compact Hausdorff spaces, then $C_{0}(Y)$ is a right $C_{0}(X)$-module with scalar multiplication $C_{0}(Y) \times C_{0}(X) \rightarrow C_{0}(Y)$ given by

$$
g \cdot f: y \mapsto g(y) f(p(y)) .
$$

If $p$ is finite, then $C_{0}(Y)$ is a Hilbert $C_{0}(X)$-module under

$$
\langle f \mid g\rangle_{C_{0}(Y)}: t \mapsto \sum_{p(y)=t} \frac{f(y)^{*} g(y)}{\left|p^{-1}(t)\right|} .
$$

Proof. The module axioms are clearly satisfied. The inner product $\langle f \mid g\rangle$ is welldefined when $p$ has finite fibres; it is continuous because $p$ is a covering, and vanishes at infinity because $f$ and $g$ do so and $p$ is finite. It is clearly sesquilinear and positive definite. We need to prove that $C_{0}(Y)$ is complete in this inner product. Let $\left\{g_{n}\right\}$ be a Cauchy sequence in $C_{0}(Y)$. Say that the fibres of $p$ have cardinality at most $N$. For $\varepsilon>0$ and large $m, n$ :

$$
\begin{aligned}
\left|g_{n}(y)-g_{m}(y)\right|^{2} & \leq \sup _{t \in X} \sum_{p(y)=t} \frac{\left|g_{n}(y)-g_{m}(y)\right|^{2}}{\left|p^{-1}(t)\right|^{2}} \\
& =\left\|\left\langle g_{n}-g_{m} \mid g_{n}-g_{m}\right\rangle_{C_{0}(Y)}\right\|_{C_{0}(X)} / N^{2}<\varepsilon
\end{aligned}
$$


for all $y \in Y$, so $\left\{g_{n}(y)\right\}$ is a Cauchy sequence in $\mathbb{C}$. Because this convergence is uniform, we obtain a continuous function $g \in C_{0}(Y)$ satisfying $g(y)=\lim g_{n}(y)$ pointwise, and hence also $\lim _{n} g_{n}=g$ in $C_{0}(Y)$.

Lemma 6.12. If $p: Y \rightarrow X$ is a finite covering between locally compact Hausdorff spaces, then the Hilbert $C(X)$-module $C(Y)$ of Example 6.11 is a nondegenerate special dagger Frobenius structure in $\mathbf{H i l b}_{C(X)}$.

Proof. As multiplication $\mu: C_{0}(Y) \otimes C_{0}(Y) \rightarrow C_{0}(Y)$, take $f \otimes g \mapsto f g$. As unit $\eta: C_{0}(X) \rightarrow C_{0}(Y)$, take $f \mapsto f \circ p$. This clearly defines a monoid. Define the counit $\gamma: C_{0}(Y) \rightarrow C_{0}(X)$ by $\gamma(g)(t)=\sum_{p(y)=t} g(y) /\left|p^{-1}(t)\right|$. Then indeed $\eta^{\dagger}=\gamma:$

$$
\langle\eta(f) \mid g\rangle_{C_{0}(Y)}(t)=\sum_{p(y)=t} \frac{\overline{f(p(y))} g(y)}{\left|p^{-1}(t)\right|}=\overline{f(t)} \sum_{p(y)=t} \frac{g(y)}{\left|p^{-1}(t)\right|}=\langle f \mid \gamma(g)\rangle_{C_{0}(X)}(t) .
$$

Then $\eta^{\dagger} \circ \eta=$ id. The pushout $C_{0}(Y) \otimes C_{0}(X) C_{0}(Y)$ of $-\circ p: C_{0}(X) \rightarrow C_{0}(Y)$ corresponds by Gelfand duality to $C_{0}\left(Y \times_{X} Y\right)$, where $Y \times_{X} Y$ is the pullback of $p$ along itself:

$$
\begin{aligned}
C_{0}(Y) \otimes C_{0}(X) & C_{0}(Y) \\
f \otimes g & \mapsto\left(\left(y_{1}, y_{2}\right) \mapsto f\left(y_{1}\right) g\left(y_{2}\right)\right) .
\end{aligned}
$$

Define the comultiplication $\delta: C_{0}(Y) \rightarrow C_{0}(Y) \otimes C_{0}(X) C_{0}(Y)$ by

$$
\delta(h)\left(y_{1}, y_{2}\right)= \begin{cases}h(y)\left|p^{-1}(p(y))\right| & \text { if } y_{1}=y_{2}, \\ 0 & \text { otherwise; }\end{cases}
$$

this is continuous because the diagonal $\Delta_{Y} \subseteq Y \times{ }_{X} Y$ is clopen by Lemma 6.10. There are $h_{i}^{(1)}, h_{i}^{(2)} \in C_{0}(Y)$ with $\delta=\sum_{i} h_{i}^{(1)} \otimes h_{i}^{(2)}$. Now we can verify that $\mu^{\dagger}=\delta$; labeling $p^{-1}(t)=\left\{y_{1}, \ldots, y_{n}\right\}$ :

$$
\begin{aligned}
\langle f \otimes g \mid \delta(h)\rangle_{C_{0}(Y) \otimes C_{0}(Y)}(t) & =\sum_{i}\left\langle f \mid h_{i}^{(1)}\right\rangle_{C_{0}(Y)}(t) \cdot\left\langle g \mid h_{i}^{(2)}\right\rangle_{C_{0}(Y)}(t) \\
& =\frac{1}{n^{2}} \sum_{j, l} \overline{f\left(y_{j}\right) g\left(y_{l}\right)} \sum_{i} h_{i}^{(1)}\left(y_{j}\right) h_{i}^{(2)}\left(y_{l}\right) \\
& =\frac{1}{n} \sum_{j} \overline{f\left(y_{j}\right) g\left(y_{j}\right)} h\left(y_{j}\right) \\
& =\frac{1}{n} \sum_{p(y)=t} \overline{f(y) g(y)} h(y) \\
& =\langle\mu(f \otimes g) \mid h\rangle_{C_{0}(Y)}(t) .
\end{aligned}
$$

Speciality $\mu \circ \mu^{\dagger}=\operatorname{id}_{C_{0}(Y)}$ is established by the following computation:

$$
\mu \circ \mu^{\dagger}(h)(y)=\left(\sum_{i} h_{i}^{(1)} h_{i}^{(2)}\right)(y)=\sum_{i} h_{i}^{(1)}(y) h_{i}^{(2)}(y)=h(y) .
$$


Next, we verify the Frobenius law:

$$
\begin{aligned}
& (\mu \otimes 1) \circ\left(1 \otimes \mu^{\dagger}\right)(f \otimes g)=(\mu \otimes 1)\left(f \otimes \sum_{i} g_{i}^{(1)} \otimes g_{i}^{(2)}\right)=\sum_{i} f g_{i}^{(1)} \otimes g_{i}^{(2)}, \\
& (1 \otimes \mu) \circ\left(\mu^{\dagger} \otimes 1\right)(f \otimes g)=(1 \otimes \mu)\left(\sum_{i} f_{i}^{(1)} \otimes f_{i}^{(2)} \otimes g\right)=\sum_{i} f_{i}^{(1)} \otimes f_{i}^{(2)} g .
\end{aligned}
$$

Under the identification $C_{0}(Y) \otimes C_{0}(X) C_{0}(Y) \simeq C_{0}\left(Y \times_{X} Y\right)$, the previous two elements of $C_{0}(Y) \otimes C_{0}(X) C_{0}(Y)$ map $\left(y_{1}, y_{2}\right) \in Y \times{ }_{X} Y$ to, respectively:

$$
\begin{aligned}
\sum_{i}\left(f g_{i}^{(1)}\right)\left(y_{1}\right) g_{i}^{(2)}\left(y_{2}\right) & =\sum_{i} f\left(y_{1}\right) g_{i}^{(1)}\left(y_{1}\right) g_{i}^{(2)}\left(y_{2}\right)=\delta_{y_{1}, y_{2}} f\left(y_{1}\right) g\left(y_{1}\right), \\
\sum_{i}\left(f_{i}^{(1)}\right)\left(y_{1}\right)\left(f_{i}^{(2)} g\right)\left(y_{2}\right) & =\sum_{i} f_{i}^{(1)}\left(y_{1}\right) f_{i}^{(2)}\left(y_{2}\right) g\left(y_{2}\right)=\delta_{y_{1}, y_{2}} f\left(y_{2}\right) g\left(y_{2}\right) .
\end{aligned}
$$

These are clearly equal to each other.

We will see in Sect. 8 below that in fact every commutative special dagger Frobenius structure in $\mathbf{H i l b}_{C_{0}(X)}$ is of the form of the previous lemma. Let us discuss two special cases to build intuition.

Example 6.13. To connect to the familiar example in FHilb, take $X=1$ and consider a two-point space $Y$ trivially covering $X$. Then the pullback $Y{ }{ }_{X} Y$ is simply the product $Y \times Y$. The Frobenius structure of the previous lemma then is $C_{0}(Y)=\mathbb{C}^{2}$. It carries the normalised version of its usual inner product. The normalisation factor is needed to make the Frobenius structure special. It is taken into the inner product, because otherwise the computations in the previous lemma involving the multiplication and comultiplication would become unreadable; the normalisation has to happen somewhere, and the inner product seems like the least objectionable place. Thus $C_{0}(Y)=\mathbb{C}^{2}$ is a Hilbert module over $C_{0}(X)=\mathbb{C}$.

Example 6.14. Applying Lemma 6.12 to the double cover of Example 6.9 with $n=2$, the pullback $Y \times_{X} Y$ is a subset $\left\{(a, b) \in S^{1} \times S^{1} \mid a^{2}=b^{2}\right\}$ of the torus. 


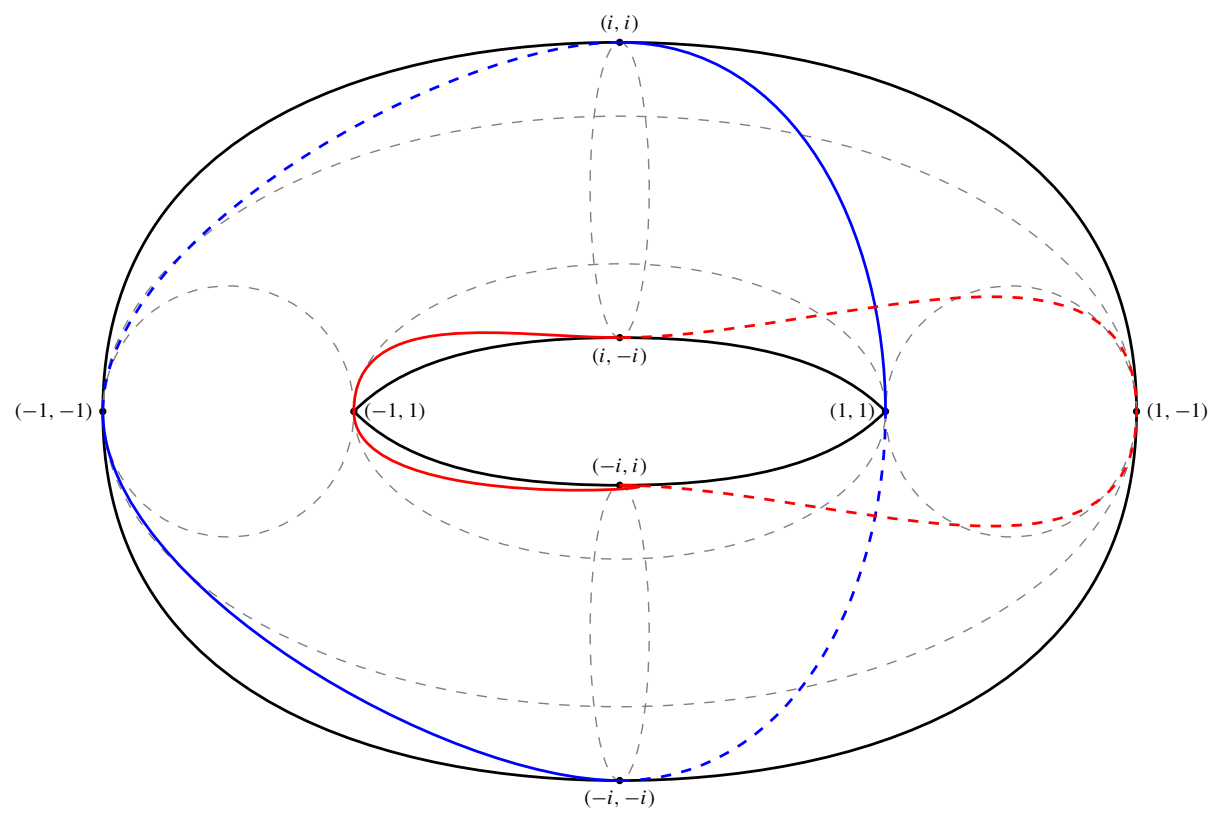

It clearly consists of two homeomorphic connected components, one of which is the diagonal $\left\{(a, a) \in S^{1} \times S^{1} \mid a \in S^{1}\right\}$, as in Lemma 6.10, and the other one is $\{(a,-a) \in$ $\left.S^{1} \times S^{1} \mid a \in S^{1}\right\}$. This enables the definition of the comultiplication $\mu^{\dagger}$ as a map of $C(X)$-modules. However, as the double cover $p$ is not trivial, it has no global sections $e_{i}$. Therefore there cannot be a description of the comultiplication $\mu^{\dagger}$ in terms of $e_{i} \mapsto e_{i} \otimes e_{i}$ as in the case $X=1$; this is only the case over local neighbourhoods of points $t \in X$.

Remark 6.15. The previous example shows that not every special dagger Frobenius structure in Hilb $_{C_{0}(X)}$ is of the form $\bigoplus \operatorname{End}\left(E_{i}\right)$ for projective Hilbert $C_{0}(X)$-modules $E_{i}$. If that were the case, since the rank of the previous example can uniquely be written as a sum of squares as $2=1+1$, then it would have to be a direct sum of two Hilbert $C_{0}(X)$-modules of rank 1 . But then it would have nontrivial idempotent central global sections, which it does not.

We end this section with nontrivial examples noncommutative special dagger Frobenius structures in $\mathbf{H i l b}_{C}$. In fact, we will consider examples that are noncommutative in an extreme sense, namely that of being central, defined as follows.

Definition 6.16. A dagger Frobenius structure $(E, \mu)$ in $\mathbf{H i l b}_{C}$ is central when $Z(E)=$ $\{x \in E \mid \forall y \in E: \mu(x \otimes y)=\mu(y \otimes x)\}=1_{E} \cdot C$ and it is faithful as a right $C$-module: $f \in C$ vanishes when $1_{E} f=0$ (or equivalently, when $x f=0$ for all $x \in E$ ).

Example 6.17. Write $\mathbb{D}=\{z \in \mathbb{C}|| z \mid \leq 1\}$ for the unit disc, $S^{1}=\{z \in \mathbb{C}|| z \mid=1\}$ for the unit circle, and $X=S^{2}=\left\{t \in \mathbb{R}^{3} \mid\|t\|=1\right\}$ for the 2-sphere. Let $n \geq 2$ be a natural number, and consider

$$
E=\left\{x \in C\left(\mathbb{D}, \mathbb{M}_{n}\right) \mid x(z)=\operatorname{diag}(\bar{z}, 1, \ldots, 1) x(1) \operatorname{diag}(z, 1, \ldots, 1) \text { if }|z|=1\right\}
$$


Then $E$ is a $C(X)$-module via the homeomorphism $X \simeq \mathbb{D} / S^{1}$; more precisely, if $q: \mathbb{D} \rightarrow X$ is the quotient map, then multiplication $E \times C(X) \rightarrow E$ is given by $(x \cdot f)(z)=x(t) \cdot f(q(t))$. Moreover, $E$ is a Hilbert $C(X)$-module under $\langle x \mid y\rangle(t)=$ $\operatorname{tr}\left(x(t)^{*} y(t)\right)$. Finally, pointwise multiplication makes $E$ a nontrivial central special dagger Frobenius structure in $\mathbf{H i l b}_{C(X)}$.

Proof. See [6, Theorem 5.8] for the fact that $E$ is the Hilbert module of sections of a nontrivial finite $C^{*}$-bundle. Use Theorem 7.7 below to see that it is a nontrivial special dagger Frobenius structure.

To see that $E$ is central, notice that

$$
\begin{aligned}
Z(E) & =\{y \in E \mid \forall x \in E \forall z \in \mathbb{D}: x(z) y(z)=y(z) x(z)\} \\
& =E \cap C\left(\mathbb{D}, Z\left(\mathbb{M}_{n}\right)\right) \\
& =E \cap C(\mathbb{D})=C(X) \cdot 1_{E}
\end{aligned}
$$

because if $y \in Z(E)$ does not take values in $Z\left(\mathbb{M}_{n}\right)$ at some $z \in \mathbb{D}$, there are two cases: if $|z|<1$ or $z=1$, then $x$ does not commute with some $y \in E$ at $z$; and if $|z|=1$, then it also does not take values in $Z\left(\mathbb{M}_{n}\right)$ at $z=1$.

\section{C*-Bundles}

We know from Sect. 4 that Hilbert modules are equivalent to Hilbert bundles. We are interested in Frobenus structures in the category of Hilbert modules, as defined in Sect. 6. In this section, we apply the bundle perspective to dagger Frobenius structures. They form $\mathrm{C}^{*}$-algebras themselves, as the following lemma shows.

Lemma 7.1. Special dagger Frobenius structures in $\mathbf{H i l b}_{C_{0}(X)}$ are $C^{*}$-algebras.

Proof. First of all, $E$ is clearly a Banach space, as an object in $\mathbf{H i l b}_{C_{0}(X)}$. It is also an algebra with multiplication $\mu: E \otimes E \rightarrow E$. In fact, it becomes a Banach algebra because $\mu^{\dagger} \mu$ is a projection by speciality [3, Lemma 9]:

$$
\begin{aligned}
\|x y\|^{2} & =\left\|\langle\mu(x \otimes y) \mid \mu(x \otimes y)\rangle_{E \otimes E}\right\|_{C_{0}(X)} \\
& =\left\|\left\langle\mu^{\dagger} \mu(x \otimes y) \mid x \otimes y\right\rangle_{E \otimes E}\right\|_{C_{0}(X)} \\
& \leq\left\|\langle x \otimes y \mid x \otimes y\rangle_{E \otimes E}\right\|_{C_{0}(X)} \\
& =\left\|\langle x \mid x\rangle_{E}\langle y \mid y\rangle_{E}\right\|_{C_{0}(X)} \\
& \leq\left\|\langle x \mid x\rangle_{E}\right\|_{C_{0}(X)}\left\|\langle y \mid y\rangle_{E}\right\|_{C_{0}(X)} \\
& =\|x\|^{2}\|y\|^{2} .
\end{aligned}
$$

Finally, this satisfies the $\mathrm{C}^{*}$-identity because it does so locally at each $t \in X$ by Lemma 6.5:

$$
\left\|x^{*} x\right\|_{E}=\sup _{t \in X}\left\|x^{*} x\right\|_{\operatorname{Loc}_{t}(E)}=\sup _{t \in X}\|x\|_{\operatorname{Loc}_{t}(E)}^{2}=\|x\|_{E}^{2} .
$$

The outer equalities use Theorem 5.6.

The $\mathrm{C}^{*}$-algebras induced by dagger Frobenius structures have more internal structure: they are in fact a bundle of $\mathrm{C}^{*}$-algebras, as made precise in the following definition. 
Definition 7.2. A finite (commutative) $C^{*}$-bundle is a bundle $p: E \rightarrow X$ where:

1. all fibres $E_{t}$ for $t \in X$ are finite-dimensional (commutative) $\mathrm{C}^{*}$-algebras;

2. any $t_{0} \in X$ has an open neighbourhood $U \subseteq X$, a finite-dimensional $\mathrm{C}^{*}$-algebra $A$, and a homeomorphism $\varphi: U \times A \rightarrow E_{U}$, such that the map $\varphi(t,-): A \rightarrow E_{t}$ is a *-isomorphism for each $t \in U$;

3. the dimension of the fibres is bounded.

If $X$ is compact, then condition (3) is superfluous.

The next lemma shows that we may view being a finite $C^{*}$-bundle as structure laid on top of being a finite Hilbert bundle.

Lemma 7.3. Any finite $C^{*}$-bundle is a finite Hilbert bundle.

Proof. Let $p: E \rightarrow X$ be a finite $\mathrm{C}^{*}$-bundle. The fibre over $t_{0} \in X$ is a finite-dimensional $C^{*}$-algebra, and hence canonically of the form $\mathbb{M}_{n_{1}} \oplus \cdots \oplus \mathbb{M}_{n_{k}}$ up to isomorphism. It is a finite-dimensional Hilbert space under the inner product

$$
\left\langle\left(a_{1}, \ldots, a_{k}\right) \mid\left(b_{1}, \ldots, b_{k}\right)\right\rangle=\operatorname{tr}\left(a_{1}^{*} b_{1}\right)+\cdots+\operatorname{tr}\left(a_{k}^{*} b_{k}\right) .
$$

Condition (2) also gives an open neighbourhood $U$ of $t_{0}$, a finite-dimensional $\mathrm{C}^{*}$-algebra $A=\mathbb{M}_{n_{1}} \oplus \cdots \oplus \mathbb{M}_{n_{k}}$, and a homeomorphism $\varphi: U \times A \rightarrow E_{U}$. Take $n=\operatorname{dim}(A)$, and let the standard matrix units constitute an orthonormal basis $e_{1}, \ldots, e_{n}$ of $A$. Define continuous sections $s_{i}: U \rightarrow E$ by $s_{i}(t)=\varphi\left(t, e_{i}\right)$. Now $\left\{s_{i}(t)\right\}$ forms an orthonormal basis of $E_{t}$ for all $t \in U$ by (2).

Example 7.4. If $X$ is a paracompact locally compact Hausdorff space, and $E$ a finitely presented projective Hilbert $C_{0}(X)$-module, then $\mathcal{L}(E)=E^{*} \otimes E \simeq \operatorname{Hilb}_{C_{0}(X)}(E, E)$ is a finite $\mathrm{C}^{*}$-bundle.

Proof. Notice that Hilb $C_{0}(X)$ is a $\mathrm{C}^{*}$-category [27, Example 1.4], and a monoidal category by Proposition 2.2. Thus it is a tensor $\mathrm{C}^{*}$-category, and hence a $2-\mathrm{C}^{*}$-category (with a single object). The result follows from [58, Proposition 2.7].

As in Sect. 4, let us spend some time on connecting to terminology in the literature. The reader only interested in new developments may safely skip may safely skip the next lemma.

Just as Definition 4.1 was a simplification of Definition 4.3, the previous definition is a simplification of the notion of field of $C^{*}$-algebras in the literature $[8,20,22,23,51,52]$ : a field $p: E \rightarrow X$ of Banach spaces where each fibre is a $\mathrm{C}^{*}$-algebra, where multiplication gives a continuous function $\left\{(x, y) \in E^{2} \mid p(x)=p(y)\right\} \rightarrow E$, and where involution gives a continuous function $E \rightarrow E$. A field of $\mathrm{C}^{*}$-algebras is uniformly finitedimensional when each fibre is finite-dimensional, and the supremum of the dimensions of the fibres is finite.

Lemma 7.5. A finite $C^{*}$-bundle is the same thing as a uniformly finite-dimensional field of $C^{*}$-algebras.

Proof. By Lemma 7.3, any finite C*-bundle is a finite Hilbert bundle, and hence a finite field of Banach spaces of locally finite rank by Lemma 4.5. Similarly, multiplication and involution are continuous functions by the same argument as in the proof of Lemma 4.5.

The converse is similar to Lemma 4.5 for the most part. Let $p: E \rightarrow X$ be a uniformly finite-dimensional field of $\mathrm{C}^{*}$-algebras. Let $t_{0} \in X$. Take $A=E_{t_{0}}$, say of the form $\mathbb{M}_{n_{1}} \oplus \cdots \oplus \mathbb{M}_{n_{k}}$, and let $x_{1}, \ldots, x_{n}$ be the orthonormal basis of $A$ constituted by 
standard matrix units. Condition (5) gives sections $s_{i}: U \rightarrow X$ with $s_{i}\left(t_{0}\right)=x_{i}$. Take $U=U_{1} \cap \cdots \cap U_{n} \cap\left\{t \in X \mid\left\{s_{i}(t)\right\}\right.$ linearly independent $\}$; this is an open subset of $X$. Define $\varphi: U \times A \rightarrow E_{U}$ by linearly extending $\left(t, s_{i}\right) \mapsto s_{i}(t)$. This is a homeomorphism, and $\varphi(t,-)$ is a $*$-isomorphism by construction.

Next, we turn to the appropriate notion of morphism between finite $\mathrm{C}^{*}$-bundles.

Definition 7.6. A morphism of finite $\mathrm{C}^{*}$-bundles is a bundle map that is fibrewise a *homomorphism. Write FCstarBundle ${ }_{X}$ for the category of finite $\mathrm{C}^{*}$-bundles with their morphisms.

We are now ready for the main result of this sectoin: to characterise the (commutative) dagger specialisable Frobenius structures in $\mathbf{H i l b}_{C_{0}(X)}$ as finite (commutative) C*-bundles over $X$.

Theorem 7.7. There is an equivalence of monoidal dagger categories

$$
\text { FCstarBundle }_{X} \stackrel{\Gamma_{0}}{\longrightarrow} \text { Frob }_{C_{0}(X)}
$$

for any paracompact locally compact Hausdorff space X.

Proof. By Proposition 6.4, we may use $\mathbf{S F r o b}_{C_{0}(X)}$ instead of $\mathbf{F r o b}_{C_{0}(X)}$. Write $\Delta$ for the adjoint of $\Gamma_{0}$ of Theorem 5.6. Let $(E, \mu, \eta)$ be a special dagger Frobenius structure in Hilb $_{C_{0}(X)}$. Equivalently, the embedding $R: E \rightarrow \mathcal{L}(E)$

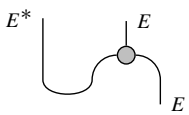

and the involution $i: E \rightarrow E^{*}$ of Eq. (2) satisfy $i \circ R=R_{*} \circ i$ [32, Corollary 9.7]. By Example 7.4, $\Delta\left(E^{*} \otimes E\right)$ is a finite $C^{*}$-bundle over $X$. Now, because both $i$ and $R$ are defined purely in terms of tensor products, composition, and dagger, the above equations also hold fibrewise by Theorem 5.6. Hence $\Delta(E)$ is a finite Hilbert bundle, which embeds into $\Delta\left(E^{*} \otimes E\right)$ with $\Delta(R)$, and is closed under the involution $\Delta(i)$. We conclude that $\Delta(E)$ is in fact a finite $\mathrm{C}^{*}$-bundle. The same reasoning establishes the converse: if $p$ is a finite $\mathrm{C}^{*}$-bundle, then $\Gamma_{0}(p)$ is a special(isable) dagger Frobenius structure in Hilb $_{C(X)}$. Compare [19, Definition 21.7]. See also [57].

The rest of this section derives from the previous theorem some corollaries of interest to categorical quantum mechanics. We start with the phase group.

Recall that the phase group of a dagger Frobenius structure $E$ consists of all morphisms $\phi: C_{0}(X) \rightarrow E$ satisfying $\left(\phi^{\dagger} \otimes \mathrm{id}\right) \circ \mu^{\dagger} \circ \phi=\eta=\left(\mathrm{id} \otimes \phi^{\dagger}\right) \circ \mu^{\dagger} \circ \phi[36]$. A group bundle is a bundle $E \rightarrow X$ whose every fibre is a group, and such that each point $t_{0} \in X$ has a group $G$ and a neighbourhood on which fibres are isomorphic to $G$. Recall that the unitary group of a unital C*-algebra $E_{t}$ is $\left\{u \in E_{t} \mid u u^{*}=u^{*} u=1\right\}$.

Corollary 7.8. The phase group of a dagger Frobenius structure $E$ in $\mathbf{F H i l b}_{C_{0}(X)}$ is a group bundle $U(E) \rightarrow X$ whose fibres are the unitary groups of fibres of $E$.

Proof. The general case follows easily from the case $X=1$, which is a simple computation [36]. 
For example, for the trivial Frobenius structure $C_{0}(X)$ in $\mathbf{F H i l b}_{C_{0}(X)}$, the phase group is the trivial bundle $U(1) \times X \rightarrow X$.

We end this section by considering a more permissive notion of morphism between Frobenius structures, namely completely positive maps.

Definition 7.9. A completely positive map between finite $\mathrm{C}^{*}$-bundles over $X$ is a bundle map that is completely positive on each fibre. Write FCstarBundle ${ }_{X}^{\mathrm{cp}}$ for the category of finite $\mathrm{C}^{*}$-bundles and completely positive maps.

In general, there is a construction that takes a monoidal dagger category $\mathbf{C}$ to a new one $\mathrm{CP}[\mathbf{C}]$, see [14]. Objects in $\mathrm{CP}[\mathbf{C}]$ are special dagger Frobenius structures in $\mathbf{C}$. Morphisms $(E, d) \rightarrow(F, d)$ in $\mathrm{CP}[\mathbf{C}]$ are morphisms $f: E \rightarrow F$ in $\mathbf{C}$ with

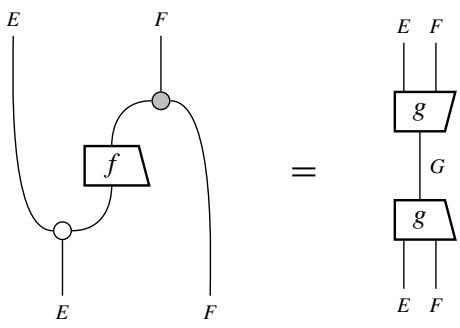

for some object $G$ and some morphism $g: E \otimes F \rightarrow G$ in $\mathbf{C}$.

Theorem 7.10. There is an equivalence of compact dagger categories

$$
\text { FCstarBundle }_{X}^{\mathrm{cp}} \stackrel{\Gamma_{0}}{\longrightarrow} \mathrm{CP}\left(\mathbf{H i l b}_{C_{0}(X)}\right)
$$

for any paracompact locally compact Hausdorff space $X$.

Proof. The correspondence on objects is already clear from Theorem 7.7. By definition, morphisms in $\mathrm{CP}\left(\mathbf{H i l b}_{C_{0}(X)}\right)$ are morphisms in $\mathbf{H i l b}_{C_{0}(X)}$ that satisfy (3). Because the equivalence is monoidal, these correspond to morphisms between finite $\mathrm{C}^{*}$-bundles that satisfy the same condition. By Theorem 7.7 the condition also holds in each fibre. Hence [14] these morphisms are completely positive maps in each fibre.

\section{Commutativity}

In this section we will completely characterise the commutative special dagger Frobenius structures in the category of Hilbert modules. By Theorem 7.7, they correspond to commutative finite $\mathrm{C}^{*}$-bundles. In this section we phrase that in terms of Gelfand duality, generalizing [43]. We first reduce to nondegenerate Frobenius structures.

Lemma 8.1. Let $X$ be a locally compact Hausdorff space. Any (specializable) dagger Frobenius structure in $\mathbf{H i l b}_{C_{0}(X)}$ is determined by a nondegenerate (specializable) one in Hilb $_{C_{0}(U)}$ for a clopen subset $U \subseteq X$.

Proof. Let $E \in \mathbf{F r o b}_{C_{0}(X)}$. By Theorem 7.7 it corresponds to a finite C*-bundle. (Note that this does not need paracompactness.) So $t \mapsto \operatorname{dim}\left(E_{t}\right)$ is a continuous function $X \rightarrow \mathbb{N}$, and $U=\left\{t \in X \mid \operatorname{dim}\left(E_{t}\right)>0\right\}$ is clopen. We need to show that the restricted finite $C^{*}$-bundle over $U$ is nondegenerate. Note that $\operatorname{dim}\left(E_{t}\right)$ is the value of the scalar $\eta^{\dagger} \circ \mu \circ \mu^{\dagger} \circ \eta \in C_{b}(X)$ at $t$. In particular, it takes values in $\mathbb{N}$, and if $t \in U$, then it is invertible. 
Next, we show that any nondegenerate specialisable dagger Frobenius structure in Hilb $_{C_{0}(X)}$ is induced by a finite bundle $p: Y \rightarrow X$.

Proposition 8.2. Let $X$ be a paracompact locally compact Hausdorff space. Any commutative nondegenerate specialisable dagger Frobenius structure in $\mathbf{H i l b}_{C_{0}(X)}$ is isomorphic as a *-algebra to $C_{0}(Y)$ for some locally compact Hausdorff space $Y$ through a finite bundle $p: Y \rightarrow X$.

Proof. By Proposition 6.4 we may assume that the given dagger Frobenius structure $E$ is special. It then follows from Lemma 7.1 that $E$ is of the form $C_{0}(Y)$ for some locally compact Hausdorff space $Y$. Applying Lemmas 3.1 and 3.4 to the unit law $\mu \circ(\eta \otimes \eta)=\eta \circ \lambda$ shows that the map $\eta: C_{0}(X) \rightarrow C_{0}(Y)$ is multiplicative. Being a morphism in $\mathbf{H i l b}_{C_{0}(X)}$ it is also additive. It preserves the involution by definition of dual objects. Hence $\eta$ is a $*$-homomorphism, which is nondegenerate as in Proposition 2.2. By Gelfand duality, therefore $\eta$ is of the form $-\circ p: C_{0}(X) \rightarrow C_{0}(Y)$ for a continuous map $p: Y \rightarrow X$. Because $\eta: C_{0}(X) \rightarrow C_{0}(Y)$ is injective by nondegeneracy, $p$ is surjective.

The complex vector space $C_{0}\left(p^{-1}(t)\right)$ contains at least as many linearly independent elements as distinct elements $y_{i}$ of $p^{-1}(t)$, namely the continuous extension of $y_{j} \mapsto \delta_{i j}$ by Tietze's extension theorem. But $C_{0}(Y)$ is finitely presented projective as a $C_{0}(X)$ module by Theorem 5.5, so there is a natural number $n$ and some $E \in$ FHilb $_{C_{0}(X)}$ such that for each $t \in X$ we have $C_{0}\left(p^{-1}(t)\right) \oplus E_{t} \simeq \mathbb{C}^{n}$ by localising as in Proposition 2.5. Thus $\operatorname{dim}\left(C_{0}\left(p^{-1}(t)\right)\right) \leq n$, and hence $p^{-1}(t)$ has cardinality at most $n$, for each $t \in X$.

Our next goal is to show that the finite bundle $p$ is of the form of Lemma 6.12. We will do this in several steps. To show that $p$ must in fact be a finite covering, we first prove $p$ is an open map.

Lemma 8.3. Let $X$ be a paracompact locally compact Hausdorff space. Nondegenerate commutative specialisable dagger Frobenius structures in $\mathbf{H i l b}_{C_{0}(X)}$ are of the form $C_{0}(Y)$ for a finite bundle $p: Y \rightarrow X$ that is open.

Proof. By Theorem 7.7 a specialisable dagger Frobenius structure $E$ in $\mathbf{H i l b}_{C_{0}(X)}$ corresponds to a finite $C^{*}$-bundle, whose fibres have uniformly bounded dimension. We need to show that $p$ is open; suppose for a contradiction that it is not. Let $V \subseteq Y$ be an open set such that $p(V) \subseteq X$ is not open. Fix a limit point $t_{0} \in p(V)$ of $X \backslash p(V)$, and pick $s_{0} \in V$ with $p\left(s_{0}\right)=t_{0}$. Urysohn's lemma now provides a continuous function $y: Y \rightarrow[0,1]$ with $y\left(s_{0}\right)=1$ that vanishes outside a compact subset of $V$ and hence vanishes at infinity. Now $\eta^{\dagger}(y)(t)=0$ if and only if $\sum_{p(s)=t} y(s)=0$ for all $t \in X$, so $\eta^{\dagger}(y)$ vanishes on $X \backslash p(V)$. But $\eta^{\dagger}(y)\left(t_{0}\right)>0$ by Lemma 8.1, contradicting continuity of $\eta^{\dagger}$. See also [43, Theorem 5.6], [9, 2.2.3] and [38, Theorem 4.3].

Next, we show that $p: Y \rightarrow X$ must also be a closed map. When $Y$ is compact and $X$ is Hausdorff this is automatic because continuous images of compact spaces are compact and compact subsets of Hausdorff spaces are closed; we show that it also holds when $Y$ is only locally compact.

Lemma 8.4. Finite bundles $p: Y \rightarrow X$ of locally compact Hausdorff spaces are closed.

Proof. Suppose $V \subseteq Y$ is closed. We want to show that $U=p(V) \subseteq X$ is closed. Let $t_{\alpha}$ be a net in $U$ that converges to $t \in X$. Pick $s_{\alpha}$ in $p^{-1}\left(t_{\alpha}\right) \cap V$. Say $p^{-1}(t) \cap V=$ $\left\{s_{1}, \ldots, s_{n}\right\}$. Pick compact neighbourhoods $V_{i} \subseteq V$ of $s_{i}$ (possible because $Y$ is locally 
compact). Then $s_{\alpha}$ is eventually in $\bigcup_{i} V_{i}$ (because this finite union is compact). So a subnet of $s_{\alpha}$ converges to one of the $s_{i} \in V$. But then, by continuity of $p$, a subnet of $t_{\alpha}$ converges to $p\left(s_{i}\right) \in U$. But then $t=p\left(s_{i}\right)$ is in $U$ (because $X$ is Hausdorff).

Finally, we can show that the $p$ must be a finite covering.

Proposition 8.5. Let $X$ be a paracompact locally compact Hausdorff space. Any nondegenerate commutative specialisable dagger Frobenius structure in $\mathbf{H i l b}_{C_{0}(X)}$ is of the form $C_{0}(Y)$ for a finite covering $p: Y \rightarrow X$.

Proof. We simplify [43, Theorem 4.4]. By Theorem 5.5, $C_{0}(Y) \oplus E \simeq C_{0}(X)^{n}$ for some $n \in \mathbb{N}$ and $E \in$ FHilb $_{C_{0}(X)}$. Hence $k_{t}=\left|\operatorname{dim}\left(C_{0}(Y)_{t}\right)\right|=\left|p^{-1}(t)\right| \leq n$ for all $t \in X$. Because $t \mapsto k_{t}$ is a continuous function $X \rightarrow \mathbb{N}$ by Remark 4.2, the subsets $X_{k}=\left\{t \in X \mid k_{t}=k\right\} \subseteq X$ are closed and open for $k=1, \ldots, n$. That is, $X=X_{1} \sqcup \cdots \sqcup X_{n}$ is a finite disjoint union of clopen subsets, on each of which the fibres of $p$ have the same cardinality.

Now for $t \in X$, by Lemma 8.4 and [43, Lemma 2.2], we can choose a neighbourhood $U \subseteq X$ over which $p^{-1}(U)$ is a disjoint union of open subsets $V_{1}, \ldots, V_{k} \subseteq Y$ that each contain a preimage of $t$. By replacing $U$ by $\bigcap p\left(V_{i}\right)$, and intersecting $V_{i}$ with $\bigcap p^{-1}\left(p\left(V_{j}\right)\right)$, we may assume that each $p: V_{i} \rightarrow U$ is surjective. But then, because all fibres have the same size, it cannot happen that one of the $V_{i}$ has two points of a fibre, as then another $V_{j}$ must have none (because there are only finitely many points in the fibre), whence $p: V_{j} \rightarrow U$ would not be surjective. So each $p: V_{i} \rightarrow U$ is a closed and open bijection, and hence a homeomorphism.

This completely characterises commutative specialisable dagger Frobenius structures in $\mathbf{H i l b}_{C_{0}(X)}$ for paracompact connected $X$. Write $\mathbf{c F r o b}_{C_{0}(X)}$ for the full subcategory of nondegenerate commutative objects in $\mathbf{F r o b}_{C_{0}(X)}$, and write Covering $\mathbf{C o r}_{X}$ for the category of finite coverings and bundle maps. The category Covering ${ }_{X}$ is symmetric monoidal under Cartesian product.

Theorem 8.6. For any paracompact locally compact Hausdorff space $X$ there is an equivalence cFrob $_{C_{0}(X)} \simeq$ Covering $_{X}$ of symmetric monoidal dagger categories.

Proof. Combine Lemmas 6.12 and 8.3 to establish the equivalence. Monoidality follows because the tensor product is the coproduct of commutative $\mathrm{C}^{*}$-algebras, and so $C_{0}(X) \otimes$ $C_{0}(Y) \simeq C_{0}(X)+C_{0}(Y) \simeq C_{0}(X \times Y)$ by duality.

Alternatively, we could include degenerate objects in $\mathbf{c F r o b}_{C_{0}(X)}$ and objects $p$ in Covering $_{X}$ to be non-surjective.

\section{Transitivity}

In this section we reduce the study of special dagger Frobenius structures to the study of central ones and commutative ones, by proving a transitivity theorem that adapts $[17$, Theorem II.3.8] to the setting of dagger Frobenius structures. We start with combining Frobenius structures $E$ over $Z$ and $Z$ over $C$ into a Frobenius structure $E$ over $C$.

Lemma 9.1. Let $C$ and $Z$ be commutative $C^{*}$-algebras with paracompact spectrum. If $E$ is a nondegenerate (specialisable) dagger Frobenius structure in $\mathbf{H i l b}_{Z}$, and $Z$ is a nondegenerate (specialisable) dagger Frobenius structure in $\mathbf{H i l b}_{C}$, then $E$ is a nondegenerate (specialisable) dagger Frobenius structure in $\mathbf{H i l b}_{C}$. 
Proof. By Theorem 7.7, there is a finite $\mathrm{C}^{*}$-bundle $p: E \rightarrow \operatorname{Spec}(Z)$, and a commutative finite $C^{*}$-bundle $Z \rightarrow X=\operatorname{Spec}(C)$. By Theorem 8.6, the latter corresponds to a branched covering $q: \operatorname{Spec}(Z) \rightarrow X$. We will show that $r=q \circ p$ is a finite $C^{*}$-bundle $E \rightarrow X$. First of all, the fibre of $r$ over $t \in X$ is $r^{-1}(t)=\bigoplus_{u \in q^{-1}(t)} p^{-1}(u)$, a finite direct sum of finite-dimensional $C^{*}$-algebras, and hence a finite-dimensional $C^{*}$-algebra. Now let $t_{0} \in X$. Say $q^{-1}\left(t_{0}\right)=\left\{u_{1}, \ldots, u_{n}\right\} \in \operatorname{Spec}(Z)$. Pick open neighbourhoods $U_{i} \subseteq \operatorname{Spec}(Z)$ of $u_{i}$, finite-dimensional C*-algebras $A_{i}$, and homeomorphisms $\varphi_{i}: U_{i} \times$ $A_{i} \rightarrow p^{-1}\left(U_{i}\right)$, such that $\varphi_{i}(u,-): A_{i} \rightarrow p^{-1}\left(U_{i}\right)$ is a $*$-isomorphism for each $u \in U_{i}$. Because $q$ is a branched covering, we may assume the $U_{i}$ disjoint. Set $V=\bigcap_{i=1}^{n} q(V)$; this is an open neighbourhood of $t_{0}$ in $X$ because $q$ is open. Set $A=\bigoplus_{i=1}^{n} A_{i}$. Define $\varphi: V \times A \rightarrow r^{-1}\left(t_{0}\right)=\bigoplus_{i=1}^{n} p^{-1}\left(U_{i}\right)$ by

$$
\varphi(t, a)=\left(\varphi_{1}\left(u_{1}, a_{1}\right), \ldots, \varphi_{n}\left(u_{n}, a_{n}\right)\right)
$$

where $a=\left(a_{1}, \ldots, a_{n}\right)$, and $t=q\left(u_{i}\right)$ for $u_{i} \in U_{i}$. Then, for each $t \in V$, say $t=q\left(u_{i}\right)$ with $u_{i} \in U_{i}$, the function

$$
\varphi(t,-)=\bigoplus_{i=1}^{n} \varphi_{i}\left(u_{i},(-)_{i}\right): A=\bigoplus_{i=1}^{n} A_{i} \rightarrow \bigoplus_{i=1}^{n} p^{-1}\left(U_{i}\right)=r^{-1}(t)
$$

is a $*$-isomorphism. It is clear that $r$ is nondegenerate when $p$ and $q$ are, and that $r$ is specialisable when $p$ and $q$ are.

The rest of this section considers the converse: if $E$ is a Frobenius structure over $C$, does it decompose into Frobenius structures $E$ over $Z$ and $Z$ over $C$ ? We start with the first step: $E$ over $Z$. Our proof below will use the following algebraic lemma.

Lemma 9.2. If $(E, \mu, \eta)$ is a specialisable dagger Frobenius structure in $\mathbf{H i l b}_{C}$, then $E=Z(E) \oplus[E, E]$ is a dagger biproduct of Hilbert modules, where $[E, E]$ is the $C$-linear span of $\{x y-y x \mid x, y \in E\}$

Proof. Adapting [4] to monoidal categories, together with the fact that specialisable Frobenius structure are symmetric, shows that $(E, \mu, \eta)$ is strongly separable [17]. By [29, Theorem 1], there is a direct sum $E \simeq Z(E) \oplus[E, E]$ of $C$-modules. It now suffices to prove that this direct sum is orthogonal, as it then follows that both summands are Hilbert modules [56, Section 15.3]. But if $z \in Z(E)$ and $x, y \in E$, then

$$
\langle z \mid x y-y x\rangle=\langle z \mid x y\rangle-\langle z \mid y x\rangle=\left\langle z y^{*} \mid x\right\rangle-\left\langle y^{*} z \mid x\right\rangle=0,
$$

where the second equation uses that dagger Frobenius structures are $\mathrm{H}^{*}$-algebras; see [2, Lemma 5], which does not depend on commutativity.

It follows that the projection $p_{1}: E \rightarrow Z(E)$ is cyclic: $p_{1}(x y)=p_{1}(y x)$. It also follows that if $E$ is a specialisable dagger Frobenius structures, its centre $Z(E)$ is a well-defined Hilbert module. We leave open the question whether special(isable) dagger Frobenius structures in arbitrary monoidal dagger categories correspond to monoidcomonoid pairs $E$ with $E \simeq Z(E) \oplus F$ a dagger biproduct, where $Z(E)$ is defined by an equaliser.

Let us consider what the centre and commutator looks like in the paradigmatic example. 
Example 9.3. Consider the special dagger Frobenius structure $E=\mathbb{M}_{n}$ in Hilb. Then $Z(E)=\mathbb{C}$, and $[E, E]=\left\{y \in \mathbb{M}_{n} \mid \operatorname{tr}(y)=0\right\}$ (see [5]) and indeed

$$
Z(E) \underset{p_{1}}{\stackrel{i_{1}}{\rightleftarrows}} E \underset{p_{2}}{\stackrel{i_{2}}{\leftrightarrows}}[E, E]
$$

forms a dagger biproduct, where $i_{1}(1)=\frac{1}{\sqrt{n}}, i_{2}(y)=y, p_{1}(x)=\frac{1}{\sqrt{n}} \operatorname{tr}(x)$, and $p_{2}(x)=x-\frac{1}{n} x$ :

$$
\begin{aligned}
\left\langle i_{1}(1) \mid x\right\rangle & =\left\langle\frac{1}{\sqrt{n}} \mid x\right\rangle=\frac{1}{\sqrt{n}} \operatorname{tr}(x)=\left\langle 1 \mid p_{1}(x)\right\rangle, \\
\left\langle i_{2}(y) \mid x\right\rangle & =\langle y \mid x\rangle=\langle y \mid x\rangle-\left\langle y \mid \frac{1}{n} \operatorname{tr}(x)\right\rangle=\left\langle y \mid p_{2}(x)\right\rangle, \\
p_{1} \circ i_{1}(1) & =p_{1}\left(\frac{1}{\sqrt{n}}\right)=\frac{1}{n} \operatorname{tr}(1)=1, \\
p_{2} \circ i_{2}(y) & =p_{2}(y)=y-\frac{1}{n} \operatorname{tr}(y)=y, \\
i_{1} \circ p_{1}+i_{2} \circ p_{2}(x) & =i_{1}\left(\frac{1}{\sqrt{n}} \operatorname{tr}(x)\right)+i_{2}\left(x-\frac{1}{n} \operatorname{tr}(x)\right)=\frac{1}{n} \operatorname{tr}(x)+x-\frac{1}{n} \operatorname{tr}(x)=x .
\end{aligned}
$$

Any special dagger Frobenius structure $E$ in $\mathbf{H i l b}_{C_{0}(X)}$ is a $\mathrm{C}^{*}$-algebra according to Lemma 7.1. Therefore so is $Z(E)$, and it makes sense to talk about the monoidal category Hilb $_{Z(E)}$.

The following two lemmas finish the proof of step one: if $E$ if Frobenius over $C$, then so is $E$ over $Z$.

Lemma 9.4. If $E$ is a special dagger Frobenius structure in $\mathbf{H i l b}_{C_{0}(X)}$, then it is also an object in Hilb $_{Z(E)}$.

Proof. First of all, $E$ is certainly a $Z(E)$-module; let us verify that it is a Hilbert $Z(E)$ module. As the inner product, take $\langle x \mid y\rangle=p_{1}\left(x^{*} y\right)$, using the projection $p_{1}: E \rightarrow$ $Z(E)$ induced by Lemma 9.2, and the involution (2). By Lemma 9.2, $p_{1}$ has norm one, and hence is a conditional expectation [53]. Thus the inclusion $p_{1}^{\dagger}: Z(E) \rightarrow E$ is a *-homomorphism, and $p_{1}$ is completely positive.

Because (completely) positive maps preserve the involution [48, p. 2], we have $\langle y|$ $x\rangle^{*}=p_{1}\left(y^{*} x\right)^{*}=p_{1}\left(x^{*} y\right)=\langle x \mid y\rangle$ for $x, y \in E$. Because $p_{1}$ is $Z(E)$-linear, also $\left\langle x \mid y+y^{\prime}\right\rangle=\langle x \mid y\rangle+\left\langle x \mid y^{\prime}\right\rangle$ and $\langle x \mid y z\rangle=\langle x \mid y\rangle z$ for $x, y, y^{\prime} \in E$ and $z \in Z(E)$. Hence the inner product is $Z(E)$-sesquilinear.

Again because $p_{1}$ is (completely) positive, $\langle x \mid x\rangle \geq 0$ for any $x \in E$. To see that the inner product is in fact positive definite, first consider the case where $X=1$ and $E=\mathbb{M}_{n}$. Then $p_{1}: \mathbb{M}_{n} \rightarrow \mathbb{C}^{n}$ takes the diagonal of a matrix. So if $x \in \mathbb{M}_{n}$, and $p_{1}\left(x^{*} x\right)=0$, then $x=0$, so certainly $p_{1}(x)=0$. This generalises to finite-dimensional $C^{*}$-algebras $E$. Next we use Proposition 2.5 to go back to the case of general $E$ : if $x \in E$ satisfies $p_{1}\left(x^{*} x\right)=0$, then for all $t \in X$ we have $\operatorname{Loc}_{t}\left(p_{1}(x)\right)=0$. So, by Theorem 4.8, in fact $p_{1}(x)=0$. Thus $\langle-\mid-\rangle$ is a well-defined $Z(E)$-valued inner product on $E$.

The inner product is complete because

$$
\|x\|_{Z(E)}^{2}=\|\langle x \mid x\rangle\|_{Z(E)}=\left\|p_{1}\left(x^{*} x\right)\right\|_{Z(E)} \leq\left\|x^{*} x\right\|_{C_{0}(X)}=\|x\|_{C_{0}(X)}^{2}
$$

by Lemma 7.1. Hence $E$ is a well-defined Hilbert $Z(E)$-module.

Lemma 9.5. If $E$ is a special dagger Frobenius structure in $\mathbf{H i l b}_{C_{0}(X)}$, then it is also a special dagger Frobenius structure in $\mathbf{H i l b}_{Z(E)}$. 
Proof. Write $C$ for $C_{0}(X)$ and $Z$ for $Z(E)$. By definition, the tensor product of $E$ with

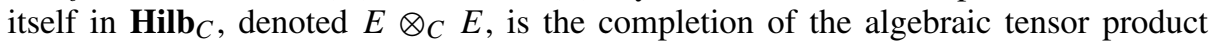
$E \odot{ }_{C} E$ in the $C$-valued inner product $\left\langle x_{1} \otimes y_{1} \mid x_{2} \otimes y_{2}\right\rangle=\left\langle x_{1} \mid x_{2}\right\rangle\left\langle y_{1} \mid y_{2}\right\rangle$. Similarly, $E \otimes_{Z} E$ is the completion of $E \odot_{Z} E$ in the $Z$-valued inner product $\left\langle x_{1} \otimes y_{1}\right|$ $\left.x_{2} \otimes y_{2}\right\rangle=p_{1}\left(x_{1}^{*} x_{2}\right) p_{1}\left(y_{1}^{*} y_{2}\right)$. The assignment $x \otimes y \mapsto x \otimes y$ extends to a canonical map $q: E \otimes_{C} E \rightarrow E \otimes_{Z} E$, because if $x_{i} \in E \odot_{C} E$ converges in the former inner product, then it does so in the latter inner product too:

$$
\left\|\left\langle x_{i} \mid x_{i}\right\rangle_{E}\right\|_{C}=\left\|x_{i}^{*} x_{i}\right\|_{C} \geq\left\|p_{1}\left(x_{i}^{*} x_{i}\right)\right\|_{Z}
$$

Here, the equality uses that (2) is a $\mathrm{C}^{*}$-involution locally as in Proposition 2.5, and the inequality uses that $p_{1}$ has norm one. Because the multiplication $\mu$ is in fact $Z$-bilinear, it factors through $q$. This gives a map $\mu_{Z}$ that makes the following diagram of modules commute.

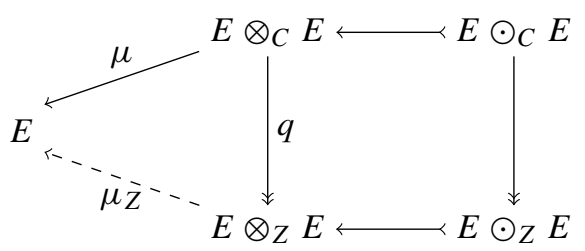

Because $\mu^{\dagger}(z x)=\mu^{\dagger} \circ \mu(z \otimes x)=\mu_{Z}\left(z \otimes \mu^{\dagger}(x)\right)=z \mu^{\dagger}(x)$ by the Frobenius law and similarly $\mu^{\dagger}(x z)=\mu^{\dagger}(x) z$, the map $\mu^{\dagger}: E \rightarrow E \otimes_{C} E$ is a morphism of $Z-Z$ bimodules. By construction $q$ is a map of $Z$-Z-bimodules. Hence $\mu_{Z}^{\dagger}=q \circ \mu^{\dagger}: E \rightarrow$ $E \otimes_{Z} E$ is $Z$-linear. Now $\left\langle x \otimes y \mid \mu_{Z}^{\dagger}(w)\right\rangle$ is computed as follows:

$$
\begin{aligned}
& \mu_{Z} \circ\left(p_{1} \otimes_{C} p_{1}\right) \circ\left(\mu \otimes_{C} \mu\right) \circ\left(\mathrm{id} \otimes_{C} \sigma \otimes_{C} \mathrm{id}\right) \circ\left(\mathrm{id} \otimes_{C} \mu^{\dagger}\right)\left(x^{*} \otimes y^{*} \otimes w\right) \\
& \quad=\mu_{Z} \circ\left(p_{1} \otimes_{C} p_{1}\right) \circ\left(\mathrm{id} \otimes_{C} \mu\right) \circ\left(\sigma \otimes_{C} \mathrm{id}\right) \circ\left(\mathrm{id} \otimes_{C} \mu^{\dagger}\right) \circ\left(\mathrm{id} \otimes_{C} \mu\right)\left(x^{*} \otimes y^{*} \otimes w\right) \\
& =\mu_{Z} \circ\left(p_{1} \otimes_{C} \mathrm{id}\right) \circ\left(\mu \otimes_{C} p_{1}\right) \circ\left(\mathrm{id} \otimes_{C} \mu^{\dagger}\right) \circ\left(\mathrm{id} \otimes_{C} \mu\right)\left(y^{*} \otimes w \otimes x^{*}\right) \\
& =\mu_{Z} \circ \mu_{Z}^{\dagger} \circ p_{1} \circ \mu \circ\left(\mathrm{id} \otimes_{C} \mu\right)\left(y^{*} \otimes w \otimes x^{*}\right) \\
& =\mu_{Z} \circ \mu_{Z}^{\dagger} \circ p_{1} \circ \mu \circ\left(\mathrm{id} \otimes_{C} \mu\right)\left(x^{*} \otimes w \otimes y^{*}\right) .
\end{aligned}
$$

This is perhaps easier to read graphically:
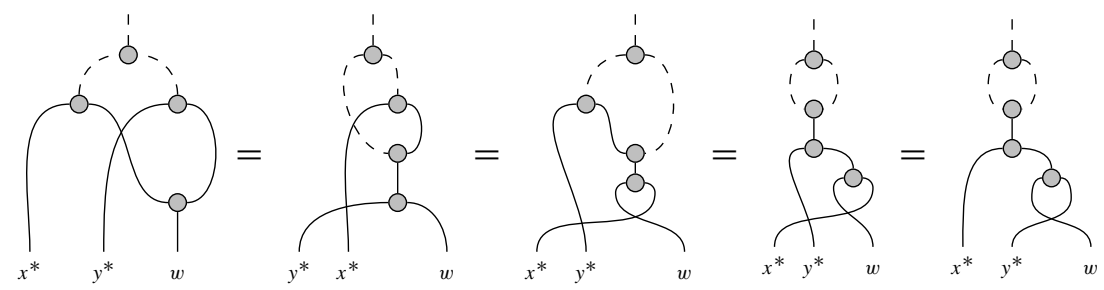

where we draw solid lines for $E$ and dashed lines for $Z$; the first and third equalities use the strong Frobenius law, and the second and fourth equalities use associativity, naturality of the swap map, and the fact that $Z$ is commutative. Thus

$$
\left\langle x \otimes y \mid \mu_{Z}^{\dagger}(w)\right\rangle=p_{1} \circ \mu_{Z} \circ\left(p_{1}^{\dagger} \otimes_{Z} p_{1}^{\dagger}\right) \circ\left(p_{1} \otimes_{Z} p_{1}\right) \circ \mu_{Z}^{\dagger}\left(y^{*} x^{*} w\right)
$$


because cyclicity of $p_{1}$ allows us to change $x^{*} w y^{*}$ into $y^{*} x^{*} w$ under this map. On the other hand, $\left\langle\mu_{Z}(x \otimes y) \mid w\right\rangle$ is $p_{1}\left(y^{*} x^{*} w\right)$. Because $\left(p_{1} \otimes_{Z} p_{1}\right) \circ \mu_{Z}^{\dagger}$ is an isometry, $p_{1}=p_{1} \circ \mu_{Z} \circ\left(p_{1}^{\dagger} \otimes_{Z(E)} p_{1}^{\dagger}\right) \circ\left(p_{1} \otimes_{Z(E)} p_{1}\right) \circ \mu_{Z}^{\dagger}$. Therefore $\mu_{Z}$ and $\mu_{Z}^{\dagger}$ are adjoints.

We can now verify the laws for special dagger Frobenius structures for $\mu_{Z}$. Unitality of $\mu_{Z}$ follows directly from unitality of $\mu$ because $\eta$ factors through $Z$. Speciality is also easy: $\mu_{Z} \circ \mu_{Z}^{\dagger}=\mu_{Z} \circ q \circ \mu^{\dagger}=\mu \circ \mu^{\dagger}=\operatorname{id}_{E}$. Now observe that $q \circ\left(\mu_{Z} \otimes_{C} \mathrm{id}_{E}\right)=$ $\left(\mu_{Z} \otimes_{Z} \mathrm{id}_{E}\right) \circ\left(\mathrm{id}_{E} \otimes_{Z} q\right)$, because both morphisms map $x \otimes y \otimes z$ to $\mu_{Z}(x \otimes y) \otimes z$. It follows from associativity of $\mu$ that

$$
\begin{aligned}
& \mu_{Z} \circ\left(\mu_{Z} \otimes_{Z} \operatorname{id}_{E}\right) \circ\left(\operatorname{id}_{E} \otimes_{Z} q\right) \circ\left(q \otimes_{C} \operatorname{id}_{E}\right) \\
& \quad=\mu_{Z} \circ\left(\operatorname{id}_{E} \otimes_{Z} \mu_{Z}\right) \circ\left(q \otimes_{Z} \operatorname{id}_{E}\right) \circ\left(\operatorname{id}_{E} \otimes_{C} q\right) .
\end{aligned}
$$

Therefore $\mu_{Z} \circ\left(\operatorname{id}_{E} \otimes_{Z} \mu_{Z}\right)$ equals $\mu_{Z} \circ\left(\mu_{Z} \otimes_{Z} \operatorname{id}_{E}\right)$ on $E \odot_{Z} E \odot_{Z} E$ and hence on all of $E \otimes_{Z} E \otimes_{Z} E$, making $\mu_{Z}$ associative. The Frobenius law follows similarly: the two morphisms

$$
\begin{aligned}
& \left(\mu_{Z} \otimes_{Z} \operatorname{id}_{E}\right) \circ\left(\operatorname{id}_{E} \otimes_{Z} \mu_{Z}^{\dagger}\right)=q \circ\left(\mu_{Z} \otimes_{C} \operatorname{id}_{E}\right) \circ\left(\operatorname{id}_{E} \otimes_{Z} \mu^{\dagger}\right) \\
& \left(\operatorname{id}_{E} \otimes_{Z} \mu_{Z}\right) \circ\left(\mu_{Z}^{\dagger} \otimes_{Z} \operatorname{id}_{E}\right)=q \circ\left(\operatorname{id}_{E} \otimes_{C} \mu_{Z}\right) \circ\left(\mu^{\dagger} \otimes_{Z} \operatorname{id}_{E}\right)
\end{aligned}
$$

equal each other on $E \odot_{Z} E$, and are therefore equal on all of $E \otimes_{Z} E$.

The last step is to prove that if $E$ is Frobenius over $C$, then so is its centre $Z(E)$.

Lemma 9.6. Let $C$ be a commutative $C^{*}$-algebra with a paracompact spectrum. If $E$ is a special dagger Frobenius structure in $\mathbf{H i l b}_{C}$, then $Z(E)$ is a specialisable dagger Frobenius structure in $\mathbf{H i l b}_{C}$.

Proof. By Theorem 7.7, $E$ corresponds to a finite $\mathrm{C}^{*}$-bundle $p: E \rightarrow X$. Define $q: Z(E) \rightarrow X$ by restriction; we will prove that it is a commutative finite $\mathrm{C}^{*}$-bundle. Clearly, $q$ is still continuous and surjective, because it maps $1 \in Z\left(E_{t}\right)$ to $t \in X$. Also, $Z(E)_{t}=Z\left(E_{t}\right)$ is a commutative finite-dimensional $\mathrm{C}^{*}$-algebra. Now let $t_{0} \in X$. Pick an open neighbourhood $U$ of $t_{0}$ in $X$, a finite-dimensional $\mathrm{C}^{*}$-algebra $A$, and a map $\varphi: U \times A \rightarrow p^{-1}(U)$ such that $\varphi(t,-): \rightarrow E_{t}$ is a $*$-isomorphism for every $t \in U$. Set $B=Z(A)$, and define $\psi: U \times B \rightarrow q^{-1}(U)=p^{-1}(U) \cap Z(E)$ to be the restriction of $\varphi$. Then $\psi(t,-): B \rightarrow q^{-1}(t)=Z(E)_{t}$ is a $*$-isomorphism.

Finally, we can state the transitivity theorem.

Theorem 9.7. Let $X$ be a paracompact locally compact Hausdorff space, and $E$ a monoid in $\mathbf{H i l b}_{C_{0}(X)}$. The following are equivalent:

(i) $E$ is a special dagger Frobenius structure in $\mathbf{H i l b}_{C_{0}(X)}$;

(ii) $E$ is a special dagger Frobenius structure in $\mathbf{H i l b}_{Z(E)}$, and

$Z(E)$ is a specialisable dagger Frobenius structure in $\mathbf{H i l b}_{C_{0}}(X)$.

Proof. Combine Lemmas 9.1, 9.5, and 9.6. The only thing left to prove is that $E$ is special over $Z(E)$ precisely when it special over $C_{0}(X)$. But this is already included in the proof of Lemma 9.5.

The latter algebra in (ii) is commutative, the former is central. We leave open the question to which monoidal dagger categories the previous theorem can be generalised [37]; there needs to be enough structure to make sense of the centre of a monoid. We also leave open the question whether it can be made functorial, that is, how the categories and Frobenius structures in (ii) of the previous theorem depend on $E$ and $X$. 


\section{Kernels}

In this final section, we return to the question of Sect. 3: what can be said about the base space given just the category of Hilbert modules? We will study special kinds of maps into the tensor unit, namely kernels. It will turn out that the existence of kernels is related to clopen subsets disconnectedness properties of the base space.

A dagger category with a zero object has dagger kernels when every morphism $f: E \rightarrow F$ has a kernel $k: K \rightarrow E$ satisfying $k^{\dagger} \circ k=\mathrm{id}_{E}$ [31]. Similarly, it has dagger equalisers when every pair of morphisms $f, g: E \rightarrow F$ has an equaliser $e$ satisfying $e^{\dagger} \circ e=$ id. In this section we show that FHilb $_{C_{0}(X)}$ has dagger kernels, and discuss when Hilb $_{C_{0}(X)}$ has dagger kernels.

Proposition 10.1. If $X$ is a locally compact Hausdorff space, Hilb ${ }_{C_{0}(X)}^{\text {bd }}$ has kernels; the kernel of $f: E \rightarrow F$ is given by (the inclusion of) $\operatorname{ker}(f)=\{x \in E \mid f(x)=0\}$.

Proof. We prove that $\operatorname{ker}(f)$ is always a well-defined object in $\mathbf{H i l b}_{C_{0}(X)}^{\text {bd }}$. The inherited inner product $\langle x \mid y\rangle_{K}=\langle x \mid y\rangle_{E}$ is still sesquilinear and positive definite. If $\left(x_{n}\right)$ is a Cauchy sequence in $\operatorname{ker}(f)$, it is also a Cauchy sequence in $E$, and hence has a limit $x \in E$. Because $f$ is adjointable, it is bounded and hence continuous, so that $f(x)=\lim _{n} f\left(x_{n}\right)=0$ and $x \in \operatorname{ker}(f)$. Thus $\operatorname{ker}(f)$ is complete.

The inclusion $\operatorname{ker}(f) \hookrightarrow E$ is bounded because it is fibrewise contractive, and hence a well-defined morphism. It inherits the universal property from the category of vector spaces.

Proposition 10.2. If $X$ is a paracompact locally compact Hausdorff space, then FHilb $_{C_{0}(X)}$ has dagger kernels; the kernel of $f: E \rightarrow F$ is given by (the inclusion of) $\operatorname{ker}(f)=\{x \in E \mid f(x)=0\}$.

Proof. First, notice that $K=\operatorname{ker}(f)$ is indeed a well-defined object of FHilb $_{C_{0}(X)}$ by Theorem 5.6: for a subbundle $\operatorname{ker}(f)$ of a finite Hilbert bundle $E$ is a finite Hilbert bundle. By Theorem 5.5, this means there exists $L \in$ FHilb $_{C_{0}(X)}$ such that $K \oplus L \simeq C_{0}(X)^{m}$ for some natural number $m$. Next, because the map $t \mapsto \operatorname{dim}\left(E_{t}\right)$ is continuous, we can write $X$ as a disjoint union of clopen subsets on which the fibres of $E$ and $F$ have constant dimension. Thus we may assume that $E=C_{0}(X)^{n}$ for some natural number

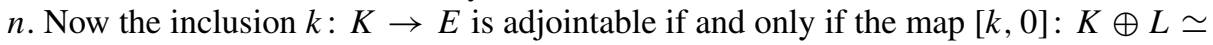
$C_{0}(X)^{m} \rightarrow C_{0}(X)^{n}$ is. But this follows from Lemma 5.2 because $k$ is bounded.

When we consider Hilbert modules that are not necessarily finitely presented projective, dagger kernels do not always exist. If they do, the base space $X$ must be totally disconnected, that is, its connected components must be singletons. If $X$ is compact this is equivalent to $C(X)$ being a $\mathrm{C}^{*}$-algebra of real rank zero.

Proposition 10.3. Let $X$ be a locally compact Hausdorff space. If $\mathbf{H i l b}_{C_{0}(X)}$ has dagger kernels, then $X$ is totally disconnected.

Proof. Let $U \subseteq X$ be a closed set containing distinct points $x, y \in X$. Since $X$ is Hausdorff, $x$ and $y$ have disjoint open neighbourhoods $V_{x}$ and $V_{y}$. Now $\{y\}$ is compact and $V_{y}$ is open, so Urysohn's lemma constructs $f \in C_{0}(X)$ with $f(y)=1$ and $f\left(X \backslash V_{y}\right)=0$ so $f(x)=0$. Regard $f$ as a morphism $C_{0}(X) \rightarrow C_{0}(X)$ by $h \mapsto f h$; it has adjoint $h \mapsto f^{*} h$. As in Lemma 3.3, $f$ has a dagger kernel of the form $K=\left\{h \in C_{0}(X) \mid h(W)=0\right\}$ for a clopen $W \subseteq X$. Now $U_{x}=U \cap(X \backslash W)$ and $U_{y}=U \cap W$ are both open in $U$, satisfy $U=U_{x} \cup U_{y}$ and $U_{x} \cap U_{y}=\emptyset$, and are not empty because $x \in U_{x}$ and $y \in U_{y}$. Therefore $U$ is not connected. That is, $X$ is totally disconnected. 
Remark 10.4. If $X$ is totally disconnected, does $\mathbf{H i l b}_{C_{0}(X)}$ have dagger kernels? The question is whether the inclusion $\operatorname{ker}(f) \hookrightarrow E$ is adjointable. The luxury of finitely presented projectivity as used in the proof of Proposition 10.2 is not available. In general it would suffice for $\operatorname{ker}(f)$ to be self-dual [41,3.3-3.4], but it is unclear whether $\operatorname{ker}(f)$ is self-dual when $E$ and $F$ are self-dual and $X$ is totally disconnected; for related functionalanalytic problems see $[24,25]$. We leave this question open.

Remark 10.5. Which categories $\mathbf{C}$ embed into $\mathbf{F H i l b}_{C_{0}(X)}$ or $\mathbf{H i l b}_{C_{0}(X)}$ for some $X$ ? We might generalise the strategy of [30, 7.2] that worked for Hilb while removing an inelegant cardinality restriction on the scalars: it suffices that $\mathbf{C}$ is symmetric dagger monoidal; has finite dagger biproducts; has dagger equalisers of cotuples $[f, g],[g, f]: E \oplus E \rightarrow$ $F$ for $f, g: E \rightarrow F$; makes every dagger monomorphism a dagger kernel; is wellpointed, and is locally small. The scalars $\mathbf{C}(I, I)$ then form a unital commutative $*$-ring, and we would need an additional condition guaranteeing that it embeds into a commutative complex *-algebra $C_{b}(X)$ for some $X$. To embed into FHilb $_{C_{0}(X)}$, we additionally require every object in the category $\mathbf{C}$ to have a dagger dual object. As a sanity check that these properties do indeed characterise categories $\mathbf{C}$ embedding into $\mathbf{F H i l b}_{C_{0}(X)}$ for some $X$, note that the category FHilb $_{C_{0}(X)}$ itself satisfies all of these properties [40, 3.6].

Acknowledgements. We thank Andreas Blass, Bertfried Fauser, Simon Henry, Klaus Keimel, and Sean Tull, and gratefully acknowledge support by EPSRC Fellowship EP/L002388/1 and NSF Grant DMS-1407152.

Open Access This article is distributed under the terms of the Creative Commons Attribution 4.0 International License (http://creativecommons.org/licenses/by/4.0/), which permits unrestricted use, distribution, and reproduction in any medium, provided you give appropriate credit to the original author(s) and the source, provide a link to the Creative Commons license, and indicate if changes were made.

\section{A. Bimodules and Bicategories}

This appendix considers the structure that results if we let the base space vary. Instead of Hilbert modules we then need to consider Hilbert bimodules, and we end up with a bicategory. It will turn out that this bicategory is a continuous extension of the wellstudied bicategory of 2-Hilbert spaces.

We start by briefly recalling Hilbert bimodules and their tensor products; for more information we refer to [40]. Recall that the adjointable maps $E \rightarrow E$ on a Hilbert $A$-module $E$ form a $\mathrm{C}^{*}$-algebra $\mathcal{L}(E)$.

Definition A.1. Let $A$ and $B$ be $C^{*}$-algebras. A Hilbert $(A, B)$-bimodule is a (right) Hilbert $B$-module $E$ together with a $*$-homomorphism $\varphi: A \rightarrow \mathcal{L}(E)$ that is nondegenerate, in the sense that $\varphi(A)(E)$ is dense in $E$. A morphism of Hilbert $(A, B)$-bimodules is an adjointable map $f: E \rightarrow F$ of (right) Hilbert $B$-modules that intertwines, i.e. $f(a(x))=a(f(x))$ for $a \in A$ and $x \in E$.

A Hilbert $\mathbb{C}$-module is simply a Hilbert space, and a morphism of $\mathbb{C}$-modules is simply an adjointable map between Hilbert spaces. A Hilbert $A$-module is the same as a Hilbert $(\mathbb{C}, A)$-bimodule, and a morphism of Hilbert $(\mathbb{C}, A)$-bimodules is the same as an adjointable map of Hilbert $A$-modules. Hence a Hilbert $(A, \mathbb{C})$-bimodule is precisely a $*$-representation of $A$, and a morphism of Hilbert $(A, \mathbb{C})$-bimodules is precisely an intertwiner. 
Definition A.2. The tensor product $E \otimes_{B} F$ of a Hilbert $(A, B)$-bimodule $E$ and a Hilbert $(B, C)$-bimodule $F$ is the algebraic tensor product of $\mathbb{C}$-modules $E \otimes_{\mathbb{C}} F$ made into a Hilbert $A-C$-bimodule under the inner product

$$
\left\langle x \otimes y \mid x^{\prime} \otimes y^{\prime}\right\rangle_{E \otimes \mathbb{C} F}=\left\langle y \mid\left\langle x \mid x^{\prime}\right\rangle_{E}\left(y^{\prime}\right)\right\rangle_{F}
$$

by quotienting out $\left\{x \in E \otimes \mathbb{C} F \mid\langle x \mid x\rangle_{E \otimes_{\mathbb{C}} F}=0\right\}$ and completing, with the map $A \rightarrow \mathcal{L}\left(E \otimes_{B} F\right)$ sending $a$ to $x \otimes y \mapsto a(x) \otimes y$.

Notice that this quotient automatically enforces $x b \otimes y=x \otimes b y$ in $E \otimes_{B} F$ for $x \in E, y \in F$, and $b \in B$. So $E \otimes_{B} F$ may alternatively be constructed as the algebraic tensor product $E \odot_{B} F$ over $B$ of $A$-B-bimodules and $B$ - $C$-bimodules by quotienting out the same subspace and completing in the same inner product.

The tensor product $E \otimes F$ of Hilbert $A$-modules $E$ and $F$ over a commutative $A$ is got by regarding them as Hilbert $(\mathbb{C}, A)$-bimodules. If $A$ is commutative, $F$ is also a Hilbert $(A, A)$-bimodule, via the map $A \rightarrow \mathcal{L}(F)$ that sends $a$ to $y \mapsto y a$. The tensor product $E \otimes_{A} F$ of Hilbert bimodules then is a Hilbert ( $\mathbb{C}, A$ )-bimodule and hence a Hilbert $A$-module $E \otimes F$. Explicitly, it is the completion of the algebraic tensor product $E \otimes_{\mathbb{C}} F$ with the following inner product and (right) $A$-module structure:

$$
\begin{aligned}
\left\langle x_{1} \otimes y_{1} \mid x_{2} \otimes y_{2}\right\rangle & =\left\langle x_{1} \mid x_{2}\right\rangle\left\langle y_{1} \mid y_{2}\right\rangle, \\
(x \otimes y) a & =x \otimes(y a) .
\end{aligned}
$$

Note that this inner product is indeed already nondegenerate [40, Proposition 4.5].

If $f: E \rightarrow E^{\prime}$ is a morphism of Hilbert $A$-B-bimodules, and $g: F \rightarrow F^{\prime}$ is a morphism of Hilbert $B$ - $C$-bimodules, then the canonical map $f \otimes_{B} g: E \otimes_{B} F \rightarrow$ $E^{\prime} \otimes_{B} F^{\prime}$ defined by $x \otimes y \mapsto f(x) \otimes g(y)$ for $x \in E$ and $y \in F$ is a well-defined morphism of Hilbert $A$ - $C$-bimodules: it is adjointable because $g$ is an intertwiner

$$
\begin{aligned}
\left\langle x^{\prime} \otimes y^{\prime} \mid f \otimes g(x \otimes y)\right\rangle_{E^{\prime} \otimes_{B} F^{\prime}} & =\left\langle x^{\prime} \otimes y^{\prime} \mid f(x) \otimes g(y)\right\rangle_{E^{\prime} \otimes_{B} F^{\prime}} \\
& =\left\langle y^{\prime} \mid\left\langle x^{\prime} \mid f(x)\right\rangle_{E^{\prime}}(g(y))\right\rangle_{F^{\prime}} \\
& =\left\langle y^{\prime} \mid g\left(\left\langle x^{\prime} \mid f(x)\right\rangle_{E^{\prime}}(y)\right)\right\rangle_{F^{\prime}} \\
& =\left\langle g^{\dagger}\left(y^{\prime}\right) \mid\left\langle f^{\dagger}\left(x^{\prime}\right) \mid x\right\rangle_{E}(y)\right\rangle_{F} \\
& =\left\langle f^{\dagger} \otimes g^{\dagger}\left(x^{\prime} \otimes y^{\prime}\right) \mid x \otimes y\right\rangle_{E \otimes_{B} F},
\end{aligned}
$$

and it is an intertwiner because $f$ is an intertwiner

$$
a(f \otimes g(x))=a(f(x)) \otimes g(y)=f(a(x)) \otimes g(y)=f \otimes g(a(x)) .
$$

Proposition A.3. There is a well-defined bicategory $\mathbf{H i l b}_{*}$ where:

- O-cells are locally compact Hausdorff spaces $X$;

- 1 -cells are Hilbert $C_{0}(X)-C_{0}(Y)$-bimodules;

- the identity 1-cell on $X$ is $C_{0}(X)$;

- horizontal composition of 1-cells is $(E, F) \mapsto E \otimes_{C_{0}(Y)} F$;

- 2-cells are morphisms of Hilbert $C_{0}(X)-C_{0}(Y)$-bimodules, i.e. adjointable intertwiners;

- the identity 2-cell on $E$ is the identity function;

- vertical composition of 2-cells is function composition;

- horizontal composition of 2-cells is $(f, g) \mapsto f \otimes_{C_{0}(Y)} g$; 
- associators $\left(E \otimes_{C_{0}(Y)} F\right) \otimes_{C_{0}(Z)} G \rightarrow E \otimes_{C_{0}(Y)}\left(F \otimes_{C_{0}(Z)} G\right)$ are given by $(x \otimes$ $y) \otimes z \mapsto x \otimes(y \otimes z)$;

- left unitors $C_{0}(X) \otimes_{C_{0}(X)} E \rightarrow E$ are given by $a \otimes x \mapsto a(x)$;

- right unitors $E \otimes_{C_{0}(Y)} C_{0}(Y) \rightarrow E$ are given by $x \otimes b \mapsto x b$;

as well as a bicategory $\mathbf{H i l b}_{*}^{\mathrm{bd}}$ where 2-cells are bounded linear intertwiners.

Proof. We have already seen that the homcategories are well-defined, and that horizontal composition is a well-defined functor. The pentagon equations are clear. The triangle equations $\left(\operatorname{id}_{E} \otimes_{C_{0}(Y)} \lambda_{F}\right) \circ \alpha_{E, C_{0}(Y), F}=\rho_{E} \otimes_{C_{0}(Y)} \mathrm{id}_{F}$ are satisfied because $x b \otimes_{C_{0}(Y)}$ $y=x \otimes_{C_{0}(Y)} b(y)$ for $b \in C_{0}(Y), x \in E$, and $y \in F$. See also [13], who use a stronger notion of 2-cells.

Notice that the endohomcategory $\mathbf{H i l b}_{*}(X, X)$ equals $\mathbf{H i l b}_{C_{0}(X)}$, so that the first (non-dagger) half of Proposition 2.2 follows from the previous one.

There is also a well-defined bicategory 2FHilb of 2-Hilbert spaces, which has as 0 -cells natural numbers, as 1-cells matrices of finite-dimensional Hilbert spaces, and as 2-cells matrices of linear maps [37,55].

Proposition A.4. There is a pseudofunctor 2FHilb $\rightarrow \mathbf{H i l b}_{*}$ that:

- sends a 0 -cell $n$ to $\{1, \ldots, n\}$;

- sends a 1-cell $\left(H_{i, j}\right): m \rightarrow n$ to $\bigoplus_{i, j} H_{i, j}$;

- sends a 2-cell $\left(f_{i, j}\right):\left(H_{i, j}\right) \rightarrow\left(K_{i, j}\right)$ to the map $\left(x_{i, j}\right) \mapsto\left(f_{i, j}\left(x_{i, j}\right)\right)$;

- is injective on 0 -cells, and a local equivalence.

Proof. Let us show that this is well-defined on 1-cells: $E=\bigoplus_{i, j} H_{i, j}$ becomes a right $\mathbb{C}^{n}$-module by $\left(x_{i, j}\right) \cdot\left(z_{j}\right)=\left(x_{i j} z_{j}\right)$; it becomes a (right) Hilbert $\mathbb{C}^{n}$-module by the inner product $\left\langle\left(x_{i, j}\right) \mid\left(y_{i, j}\right)\right\rangle_{E}(j)=\sum_{i}\left\langle x_{i, j} \mid y_{i, j}\right\rangle_{H_{i, j}}$; it becomes a Hilbert $\mathbb{C}^{m}-\mathbb{C}^{n}$ bimodule by the $*$-representation $\mathbb{C}^{m} \rightarrow \mathcal{L}(E)$ sending $\left(z_{i}\right)$ to $\left(x_{i, j}\right) \mapsto\left(z_{i} x_{i, j}\right)$.

It is also well-defined on 2-cells: the map $f: x_{i, j} \mapsto\left(f_{i, j}\left(x_{i, j}\right)\right)$ is adjointable because $\sum_{i}\left\langle f_{i, j}\left(x_{i, j}\right) \mid y_{i, j}\right\rangle_{K_{i, j}}=\sum_{i}\left\langle x_{i, j} \mid f_{i, j}^{\dagger}\left(y_{i, j}\right)\right\rangle_{H_{i, j}}$; and it is intertwining because $f_{i, j}\left(z_{i} x_{i, j}\right)=z_{i} f_{i, j}\left(x_{i, j}\right)$. This is clearly functorial on homcategories.

The pseudofunctorial data consists of 2-cells $\mathbb{C}^{n} \rightarrow \bigoplus_{i, j=1}^{n} \delta_{i, j} \mathbb{C}$ for identities, and $\left(\bigoplus_{a, b} H_{a, b}\right) \otimes_{\mathbb{C}^{n}}\left(\bigoplus_{c, d} K_{c, d}\right) \rightarrow \bigoplus_{i, j, k} H_{i, k} \otimes K_{k, j}$ for composition. By construction $\left(\bigoplus_{a, b} H_{a, b}\right) \otimes \mathbb{C}^{n}\left(\bigoplus_{c, d} K_{c, d}\right)$ is $\bigoplus_{a, b, c, d} H_{a, b} \otimes K_{c, d}$, where we identify $\left(\left(x_{a, b}\right) \otimes\right.$ $\left.\left(y_{c, d}\right)\right)$ with 0 when $x_{a, b} y_{b, d}=0$ for all $a$ and $d$. Hence there are natural candidates for both, that are adjointable intertwiners, and furthermore are in fact unitary. The coherence diagrams clearly commute.

Finally, this pseudofunctor is clearly injective on 0 -cells, and moreover, it is an equivalence on homcategories; see also [10, Proposition 8.1.11].

Thus 2FHilb is a full subcategory of $\mathbf{H i l b}_{*}$. In other words, $\mathbf{H i l b}_{*}$ is a conservative infinite continuous extension of the finite discrete 2 FHilb that is more suitable for local quantum physics.

\section{B. Complete Positivity}

Localization, as discussed in Sect. 2, is essential to the theory of Hilbert modules. And conditional expectations are essential to localization. They are a certain kind of completely positive map. In this appendix we study the category of commutative $\mathrm{C}^{*}$-algebras 
and completely positive maps further. Write $\mathbf{c C s t a r}_{\mathrm{cp}}$ for the category of commutative $\mathrm{C}^{*}$-algebras and (completely) positive linear maps. By Gelfand duality, its objects are isomorphic to $C_{0}(X)$ for locally compact Hausdorff spaces $X$. We now consider morphisms.

Definition B.1. A Radon measure on a locally compact Hausdorff space $X$ is a positive Borel measure $\mu$ satisfying $\mu(U)=\sup _{K \subseteq U} \mu(K)$ where $K$ ranges over the compact subsets of open sets $U$. Write $\operatorname{Radon}(X)$ for the set of Radon measures on $X$.

The set $\operatorname{Radon}(X)$ becomes a locally compact Hausdorff space [50, Chapter 13] under the following, so-called vague, topology: a net $\mu_{n}$ converges to $\mu$ if and only if $\int_{X} f \mathrm{~d} \mu_{n}$ converges to $\int_{X} f \mathrm{~d} \mu$ for all measurable $f: X \rightarrow \mathbb{C}$.

Definition B.2. Write Radon for the following category.

- Objects are locally compact Hausdorff spaces $X$.

- Morphisms $X \rightarrow Y$ are continuous functions $X \rightarrow \operatorname{Radon}(Y)$.

- Composition of $f: X \rightarrow \operatorname{Radon}(Y)$ and $g: Y \rightarrow \operatorname{Radon}(Z)$ is given by

$$
(g \circ f)(x)(U)=\int_{Y} g_{U} \mathrm{~d} f(x)
$$

where $g_{U}: Y \rightarrow \mathbb{C}$ for measurable $U \subseteq Z$ is defined by $y \mapsto g(y)(U)$.

- The identity on $X$ sends $x$ to the Dirac measure $\delta_{x}$.

Proposition B.3. There is an equivalence of categories

$$
\begin{aligned}
F: \text { Radon } & \rightarrow \text { cCstar }_{\mathrm{cp}}^{\mathrm{p}} \\
F(X) & =C_{0}(X) \\
F(f)(h)(x) & =\int_{X} h \mathrm{~d} f(x) .
\end{aligned}
$$

Proof. The proof of [26, Theorem 5.1] shows that $F(X)=C_{0}(X)$ and $F(f)(h)(x)=$ $f(x)(h)$ define an equivalence $F: \mathbf{R} \rightarrow \mathbf{C s t a r}_{\mathrm{cp}}^{\mathrm{op}}$, for the following category $\mathbf{R}:$

- Objects are locally compact Hausdorff spaces $X$.

- Morphisms $X \rightarrow Y$ are continuous maps $X \rightarrow R(Y)=\operatorname{cCstar}_{\mathrm{cp}}\left(C_{0}(Y), \mathbb{C}\right)$.

- Composition of $f: X \rightarrow R(Y)$ and $g: Y \rightarrow R(Z)$ is given by

$$
(g \circ f)(x)(\varphi)=f(x)\left(\mathrm{ev}_{\varphi} \circ g\right)
$$

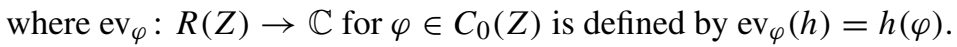

- The identity on $X$ sends $x$ to the map $C_{0}(X) \rightarrow \mathbb{C}$ defined by $k \mapsto k(x)$.

But every element of $R(X)$ is of the form $\int_{X}(-) \mathrm{d} \mu$ for a unique $\mu \in \operatorname{Radon}(X)$ (see [46, Theorem 2.14]), translating to the statement of the proposition.

Finally we consider the special case of conditional expectations.

Proposition B.4. The wide subcategory Cstar $_{\mathrm{cp}}$ of conditional expectations is dually

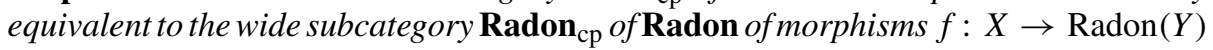
with a continuous surjection $g: Y \rightarrow X$ satisfying $\operatorname{supp}(f(x)) \subseteq g^{-1}(x)$.

Proof. Simply restrict the equivalence of Proposition B.3. Concretely, a morphism $(f, g)$ of Radon $_{\mathrm{cp}}$ gets sent to the following conditional expectation: the injective $*-$ homomorphism is $-\circ g: C_{0}(X) \longmapsto C_{0}(Y)$, and the completely positive map $C_{0}(Y) \rightarrow$ $C_{0}(X)$ maps $\varphi \in C(Y)$ to the function $x \mapsto \int_{Y} g \mathrm{~d} f(x)$. Conversely, a conditional expectation $E$ is sent to the unique morphism $(f, g)$ satisfying $E(\varphi)(y)=\int_{X} \varphi \mathrm{d} g(f(y))$. See also [44, Theorem 5.3.3]. 


\section{References}

1. Abramsky, S., Brandenburger, A.: The sheaf-theoretic structure of non-locality and contextuality. New J. Phys. 13, 113036 (2011)

2. Abramsky, S., Heunen, C.: $\mathrm{H}^{*}$-algebras and nonunital Frobenius algebras: first steps in infinitedimensional categorical quantum mechanics. AMS Proc. Symp. Appl. Math. Clifford Lect. 71, 124 (2012)

3. Abramsky, S., Heunen, C.: Logic and algebraic structures in quantum computing and information. Chapter Operational Theories and Categorical Quantum Mechanics. Cambridge University Press, Cambridge (2015)

4. Aguiar, M.: A note on strongly separable algebras. Bol. Acad. Nac. Ciencias 65, 51-60 (2000)

5. Albert, A.A., Muckenhoupt, B.: On matrices of trace zero. Mich. Math. J. 4(1), 1-3 (1957)

6. Antonevich, A., Krupnik, N.: On trivial and non-trivial $n$-homogeneous $\mathrm{C}^{*}$-algebras. Integr. Equ. Oper. Theory 38, 172-189 (2000)

7. Auslander, M., Goldman, O.: The Brauer group of a commutative ring. Trans. Am. Math. Soc. 97, 367409 (1960)

8. Blackadar, B.: Operator Algebras: Theory of $\mathrm{C}^{*}$-Algebras and Von Neumann Algebras. Springer, Berlin (2006)

9. Blanchard, E., Kirchberg, E.: Global Glimm halving for C*-bundles. J. Oper. Theory 52, 385-420 (2004)

10. Blecher, D.P., Le Merdy, C.: Operator Algebras and Their Modules, an Operator Space Approach. Oxford University Press, Oxford (2004)

11. Blute, R., Comeau, M.: Von Neumann categories. Appl. Categ. Struct. 23(5), 725-740 (2015)

12. Bos, R.: Continuous representations of groupoids. Houst. J. Math. 37(3), 807-844 (2011)

13. Buss, A., Zhu, C., Meyer, R.: A higher category approach to twisted actions on $C^{*}$-algebras. Proc. Edinb. Math. Soc. 56, 387-426 (2013)

14. Coecke, B., Heunen, C., Kissinger, A.: Categories of quantum and classical channels. Quantum Information Processing (2014)

15. Coecke, B., Lal, R.: Causal categories: relativistically interacting processes. Found. Phys. 43(4), 458501 (2012)

16. Dauns, J., Hofmann, K.H.: Representation of Rings by Sections. American Mathematical Society, Providence (1968)

17. DeMeyer, F., Ingraham, E.: Separable algebras over commutative rings. Number 181 in Lecture Notes in Mathematics. Springer, Berlin (1971)

18. Dixmier, J.: C*-algebras. North Holland (1981)

19. Dixmier, J., Douady, A.: Champs continues d'espaces Hilbertiens et de C*-algèbres. Bull. Soc. Math. Fr. 91, 227-284 (1963)

20. Dupré, M.J.: Classifying Hilbert bundles. J. Funct. Anal. 15(3), 244-278 (1974)

21. Enrique Moliner, P., Heunen, C., Tull, S.: Space in monoidal categories. Quantum Phsyics and Logic (2017). arXiv: 1704.08086

22. Fell, J.M.G.: An extension of Mackey's method to Banach *-algebraic bundles. Number 90 in Memoirs. American Mathematical Society, Providence (1969)

23. Fell, J.M.G., Doran, R.S.: Representations of *-algebras, locally compact groups, and Banach *-algebraic bundles. Number 125, 126 in Pure and Applied Mathematics. Academic, Berlin (1988)

24. Frank, M.: Hilbert $C^{*}$-modules over monotone complete $C^{*}$-algebras. Math. Nachr. 175, 61-83 (1995)

25. Frank, M., Paulsen, V.: Injective and projective Hilbert $C^{*}$-modules, and $C^{*}$-algebras of compact operators (2006). arXiv:math.OA/0611348

26. Furber, R.W.J., Jacobs, B.P.F.: From Kleisli categories to commutative C*-algebras: probabilistic Gelfand duality. Log. Methods Comput. Sci. 11(2), 5 (2015)

27. Ghez, P., Lima, R., Roberts, J.E.: $W^{*}$-categories. Pac. J. Math. 120, 79-109 (1985)

28. Gogioso, S., Genovese, F.: Infinite-dimensional categorical quantum mechanics. In: Quantum Physics and Logic (2016)

29. Hattori, A.: On strongly separable algebras. Osaka J. Math. 2, 369-372 (1965)

30. Heunen, C.: An embedding theorem for Hilbert categories. Theory Appl. Categ. 22(13), 321-344 (2009)

31. Heunen, C., Jacobs, B.: Quantum logic in dagger kernel categories 27(2), 177-212 (2010)

32. Heunen, C., Karvonen, M.: Monads on dagger categories. Theory Appl. Categ. 31(35), 1016-1043 (2016)

33. Heunen, C., Kissinger, A.: Can quantum theory be characterized in terms of information-theoretic constraints? (2016). arXiv:1604.05948

34. Heunen, C., Kissinger, A., Selinger, P.: Completely positive projections and biproducts. In: Quantum Physics and Logic X, Number 171 in Electronic Proceedings in Theoretical Computer Science, pp. 71-83 (2014)

35. Heunen, C., Tull, S.: Categories of relations as models of quantum theory. In: Quantum Physics and Logic XII, Number 195 in Electronic Proceedings in Theoretical Computer Science, pp. 247-261 (2015) 
36. Heunen, C., Vicary, J.: Categories for Quantum Theory: An Introduction. Oxford University Press, Oxford (2017)

37. Heunen, C., Vicary, J., Wester, L.: Mixed quantum states in higher categories. In: Quantum Physics and Logic, volume 172 of Electronic Proceedings in Theoretical Computer Science, pp. 304-315 (2014)

38. Ivankov, P.: Quantization of noncompact coverings (2017). arXiv:1702.07918

39. Kelly, G.M., Laplaza, M.L.: Coherence for compact closed categories. J. Pure Appl. Algebra 19, 193213 (1980)

40. Lance, E.C.: Hilbert $C^{*}$-modules, volume 210 of London Mathematical Society Lecture Note Series. Cambridge University Press, Cambridge (1995). A toolkit for operator algebraists

41. Paschke, W.L.: Inner product modules over B*-algebras. Trans. Am. Math. Soc. 182, 443-468 (1973)

42. Paulsen, V.: Completely Bounded Maps and Operator Algebras. Cambridge University Press, Cambridge (2002)

43. Pavlov, A.A., Troitskii, E.V.: Quantization of branched coverings. Russ. J. Math. Phys. 18(3), 338352 (2011)

44. Pluta, R.: Ranges of Bimodule Projections and Conditional Expectations. Cambridge Scholars, Cambridge (2013)

45. Raeburn, I., Williams, D.P.: Morita Equivalence and Continuous-Trace C*-Algebras, volume 60 of Mathematical Surveys and Monographs. American Mathematical Society, Providence (1998)

46. Rudin, W.: Real and Complex Analysis, 3rd edn. McGraw-Hill, New York (1987)

47. Selinger, P.: A survey of graphical languages for monoidal categories. In: New Structures for Physics, Lecture Notes in Physics, pp. 289-355. Springer, Berlin (2009)

48. Størmer, E.: Positive Linear Maps of Operator Algebras. Springer, Berlin (2013)

49. Takahashi, A.: Hilbert modules and their representation. Rev. Columbiana Mat. 13, 1-38 (1979)

50. Taylor, M.E.: Measure Theory and Integration. American Mathematical Society, Providence (2006)

51. Tomiyama, J.: Topological representation of $C^{*}$-algebras. Tohoku Math. J. 14(2), 187-204 (1962)

52. Tomiyama, J., Takesaki, M.: Applications of fibre bundles to the certain class of $\mathrm{C}^{*}$-algebras. Tohoku Math. J. 13(3), 498-522 (1961)

53. Tomiyama, Y.: On the projection of norm one in $W^{*}$-algebras. Proc. Jpn. Acad. 33(10), 608-612 (1957)

54. Vicary, J.: Categorical formulation of quantum algebras. Commun. Math. Phys. 304(3), 765-796 (2011)

55. Vicary, J.: Higher quantum theory (2012). arXiv: 1207.4563

56. Wegge-Olsen, N.E.: K-Theory and C*-Algebras. Oxford University Press, Oxford (1993)

57. Yamagami, S.: Frobenius duality in $C^{*}$-tensor categories. J. Oper. Theory 52, 3-20 (2004)

58. Zito, P.A.: 2-C*-categories with non-simple units. Adv. Math. 210(1), 122-164 (2007) 